\title{
Post-disaster assessment routing problem
}

\author{
Buse Eylul Oruc, Bahar Yetis Kara* \\ Department of Industrial Engineering, Bilkent University, Ankara, 06800 Turkey
}

\section{A R T I C L E I N F O}

\section{Article history:}

Received 12 February 2018

Revised 2 August 2018

Accepted 3 August 2018

Available online 11 August 2018

\section{MSC:}

00-01

99-00

Keywords:

Disaster management

Humanitarian logistics

General routing

Multi-objective

\begin{abstract}
A B S T R A C T
In this study, we propose a post-disaster assessment strategy as part of response operations in which effective and fast relief routing are of utmost importance. In particular, the road segments and the population points to perform assessment activities on are selected based on the value they add to the consecutive response operations. To this end, we develop a bi-objective mathematical model that provides damage information in the affected region by considering both the importance of population centers and road segments on the transportation network through using aerial and ground vehicles (drones and motorcycles). The first objective aims to maximize the total value added by the assessment of the road segments (arcs) whereas the second maximizes the total profit generated by assessing points of interests (nodes). Bi-objectivity of the problem is studied with the $\epsilon$-constraint method. Since obtaining solutions as fast as possible is crucial in the post-disaster condition, heuristic methods are also proposed. To test the mathematical model and the heuristic methods, a data set belonging to Kartal district of Istanbul is used. Computational experiments demonstrate that the use of drones in post-disaster assessment contributes to the assessment of a larger area due to its angular point of view. Also, the proposed heuristic methods not only can find a high-quality approximation of the Pareto front but also mitigates the solution time difficulties of the mathematical model.
\end{abstract}

(c) 2018 Elsevier Ltd. All rights reserved.

\section{Motivation and problem definition}

In the course of the last 70 years, disasters have grown exponentially both in number and magnitude (Ozdamar and Ertem, 2015). As put forward by the International Federation of Red Cross and Red Crescent Societies (IFRC) in the 2016 World Disasters Report, "humanitarian needs are growing at an extraordinary pace - a historical pace - and are outstripping the resources that are required to respond." (IFRC, 2016).

Humanitarian logistics which compromise of logistics activities while focusing on alleviating the suffering of vulnerable people is considered as one of the imperfect areas to invest in for both academics and practitioners (Kovács and Spens, 2007). In that framework, as part of humanitarian logistics, Disaster Operations Management (DOM), is defined as activities that are performed before, during, and after a disaster to prevent loss of human life, reduce its impact, and regain the normalcy (Altay and Green, 2006). The life cycle of disaster operations is divided into three categories, pre-disaster, response and recovery operations. Pre-disaster operations -mitigation and preparedness- include taking measures to avoid disaster or to reduce the impact and to gain the ability to respond to the disaster. Response is the stage where resources are utilized to reach the disaster area, save lives and prevent further damage. Recovery activities are post-disaster opera-

\footnotetext{
* Corresponding author.

E-mail addresses: buseeyluloruc@bilkent.edu.tr (B.E. Oruc), bkara@bilkent.edu.tr (B.Y. Kara).
} 
tions that aim to re-establish a normal state. Although measures and precautions are taken, disasters are not preventable and predictable. Thus, planning disaster relief operations in advance, and implementing them in disaster and post-disaster phases are significant to mitigate the destructive impact of disasters.

In case of disasters, availability of shelter, food, and water may be disrupted and even worse, people may be in need of urgent medical attention. Therefore, after disasters, logistics operations need to be conducted mainly for providing relief goods, such as food, and shelter to the disaster-affected regions, evacuating people from the danger zones, alleviating human suffering, and most importantly, saving lives. Having capable resources to handle the situation and reaching and activating them on time to alleviate the disaster impact on population and infrastructure are some of the challenges of humanitarian disaster relief operations. Moreover, logistics operations often have to be carried out in an environment with destructed transportation infrastructures (Long, 1997). Disrupted roads and debris blocking the roads are main sources of difficulty in terms of both aid distribution to disaster victims and re-establishing normal state in disaster-affected areas. In addition, the unpredictable nature of the disaster and demand uncertainty may complicate handling and distribution operations. In that perspective, assessing damage at early stages of the disaster plays a crucial role in the activation of resources.

The damage assessment module of any disaster should include the information on the death toll, location of casualties, and the extent of damage to roads, arteries and critical facilities like hospitals and schools. The information for these can be collected from various channels, which may include mobile teams, drones, and various other reports. The information collected allows disaster management operation coordinators to determine immediate actions necessary to respond to the effects of the damage with the effective use of resources.

Damage assessment can be divided into two categories based on its focus; it could focus on areas with the concentrated population (node module) and the road segments connecting them (arc module). Efficient disaster management operations should consider both elements of damage assessment simultaneously. In that perspective, post-disaster assessment operations should mainly concentrate on assessment of critical population points and critical road segments. Densely populated population points are candidates for critical and should be prioritized. Early assessment of those points results with a better understanding of essential needs such as the number of vehicles for evacuation, the number of ambulances/search and rescue teams to be dispatched or any type of relief items and their quantities.

Besides the assessment of critical points, ground network conditions have to be assessed in order to determine the available transportation routes and the roads that have to be unblocked by removing debris. The critical points, such as hospitals and schools, should remain accessible by the disaster victims. Furthermore, critical points may be in need of emergency relief item supply. Hence, to be able to maintain access to these points, assessing the disaster impact on the ground transportation network is important. The two components of damage assessment are complementary; therefore, both of them should be taken into account simultaneously during disaster assessment phase.

The main purpose of this paper is to provide a framework that considers early damage assessment regarding the severity of the disaster impact and the urgency of the need for relief on road network and population areas. The reason for the early damage assessment is to find the most effective strategy for further disaster operations. Also, to ensure the connectivity of disaster network, by estimating the amount of debris on the roads, immediate debris removal actions can be determined to unblock the disrupted road segments. Since damage assessment operations must be completed quickly, the assessment teams are not required to assess all of the affected regions and the transportation network. Therefore the population points and the roads to be assessed are selected based on their importance in the network.

In this study, we focus on developing a systematic method that can be used by municipalities or local relief agencies to determine disaster impact on their region. We assume that the critical network elements of the area are known. The criticality of population points and road segments are determined by the amount of population and the related distances. The assessment teams like motorcycles and/or drones assumed to be present at potential starting points, the depots. As there will possibly be debris or destruction on the roads, post-disaster transportation network is considered to be off-road. It is assumed that the motorcycles can only conduct an assessment of the road segments and points that lie in their paths. Whereas, as drones can fly at certain altitudes, flying over certain road segments with drones will enable the assessment of other roads and nodes in their point of view. The vehicles start their tours just after the disaster hits and they assess critical population centers and critical roads in the predetermined time frame and after the vehicles complete their tours, disaster information is reported to the depots (disaster management centers). Then, given the set of importance carried by each network element and the assumptions, we define the Post-disaster Assessment Routing Problem (PDARP) that determines: (i) the population points to visit, (ii) road segments to traverse, and (iii) the vehicle routes while considering maximum assessment in (i) and (ii) within the assessment period.

As we aim to have information on both arcs and nodes, the problem can be considered as a variant of the General Routing Problem (GRP) with profits. Aiming to assess critical population points may hinder the assessment of the critical roads in a given time period. On the other hand, aiming to assess the critical roads in limited time may result in an assessment of lesser population points but assessing/visiting them multiple times. Due to the nature of the problem, monitoring critical nodes and critical arcs at the same time, the standard requirement of the classical routing problems, that each node is to be visited exactly once, is no longer valid. Allowing multiple node passages, combining two objectives in a bi-objective manner, utilizing a heterogeneous set of vehicles and enabling a wider view, raise a new problem that we refer as Post-Disaster Assessment Routing Problem (PDARP).

In this study, we propose a different modelling perspective for the post-disaster assessment problem. We consider the assessment of population points and road segments through utilizing a heterogeneous set of vehicles, motorcycles, and drones 
which can provide a wider point of view. Allowing node/arc passages multiple times, which can be helpful in capturing the extend of damage in the disaster aftermath, is another special feature of the model proposed. By developing a mathematical model for PDARP and by using real data from Istanbul, we highlight the importance of considering both network elements and using drones and motorcycles for developing an appropriate assessment strategy.

\section{Related literature review}

As our study revolves around relief routing and assessment, the primary focus will be on those studies in the literature review. The relief routing models will be categorized according to the application areas and the problem characteristics. Then, PDARP's connections to GRP and its variants will be reviewed with a focus on the pioneering works. Finally, we will consider drone applications in routing/delivery and data acquisition.

\subsection{Relief routing literature}

Especially with the beginning of the 21st century, the increase in attention to humanitarian logistics by both academics and practitioners is followed by an increase in the number of studies (Kovács and Spens, 2007). Hence, various literature reviews are conducted on humanitarian logistics. Altay and Green (2006), Galindo and Batta (2013), Kovács and Spens (2007) and Celik et al. (2012) evaluate disaster management and relief operations literature, respectively, based on disaster timeline, types and application areas together with the solution methodologies. Caunhye et al. (2012) categorize optimization problems arise in the emergency logistics in terms of objectives, prominent constraints, and decisions they make. Further, Celik et al. (2012) provide case studies to reflect the important aspect of the different humanitarian problems. The survey conducted by Ozdamar and Ertem (2015) includes the models of response and recovery planning phases of disaster with the information system applications. Most recently, Kara and Savaser (2017) survey operations research (OR) problems encountered in the relief and development logistics.

Relief routing literature mainly focused on evacuation problems, relief item distribution, and debris removal problems. Evacuation problems focus on the safe and rapid transfer of disaster-affected people to the healthcare centers and shelters (Bayram and Yaman, 2015). Relief item distribution problem aims to find an efficient and effective distribution of pre-positioned relief items to people in need. Campbell et al. (2008), Houming et al. (2008), Ozkapici et al. (2016) tackle minimization of total delivery time or latest arrival of a vehicle in a deterministic setting. Camacho-Vallejo et al. (2015); Tzeng et al. (2007) consider minimization of the cost of most efficient relief item distribution while considering cost and fairness, respectively. Besides relief item distribution, Yan and Shih (2009) incorporate emergency road repair to the problem and Ozdamar (2011) studies relief item distribution and evacuation at the same time with helicopters.

Debris removal aspect of relief logistics literature considers reaching critical nodes and restoring network connectivity. Sahin et al. (2016) and Berktas et al. (2016) route debris removal vehicles to assure accessibility to critical points like hospitals and schools after an earthquake. Akbari and Salman (2017) work on the post-earthquake network to sustain the connectivity in a short period of time. Hua and Sheu (2013) aim to remove debris with the least cost. Celik et al. (2015) study the debris clearance problem in a stochastic setting and the aim is to maximize the total satisfied demand.

From these studies, we observe that although the damage on roads and the needs of disaster victims are considered in some relief routing problems, collecting information about the extent of damage is not received much attention. Although need assessment problem is investigated by Tatham (2009), it is not covered in an OR context. In some studies, needs assessment of disaster victims is conducted using sampling techniques. Johnson and Wilfert (2008) use cluster sampling technique which divides the disaster-affected region into disjoint clusters. In Daley et al. (2001), geography-based sampling scheme is provided. Huang et al. (2013) determine the routes for vehicles to assess needs of all communities in a disaster region such that the total arrival times is minimized via continuous approximation. A recent study of Balcik (2017) considers needs assessment of community groups where communities to conduct assessment are selected based on community characteristics using purposive sampling. In that study, routing policies are developed such that each community group and each arc can be traversed at most once by each team. The study of Balcik (2017) is the closest relative to PDARP in the humanitarian logistics domain that develops routing strategies and selects communities to assess. The problem discussed in Balcik (2017) and PDARP differ in the objectives and assumptions. While Balcik (2017) focuses on monitoring disaster impact on population centers, assuming each community/road can be visited at most once, in this paper, we relax that assumption and provide an assessment strategy that focuses both on population points and road segments.

\subsection{General routing literature}

The GRP aims to find a least-cost route that starts and ends at the same node and visits the required nodes by traversing through the required edges at least once (Orloff, 1974). There is a variant of GRP -Undirected Capacitated GRP with Profits (UCGRP with profits) (Archetti et al., 2017) and Bus Touring Problem (BTP) (Deitch and Ladany, 2000)- that does not have required nodes or edges to be traversed. In UCGRP with profits, there is a fleet of homogeneous vehicles to serve the customers which are located on nodes and edges of the network. Customers to serve; i.e. nodes and edges to traverse are selected based on maximizing the difference between the profit gained by traversing nodes and edges, and cost of traversal. UCGRP with profits can be considered as a bi-objective; however, its bi-objectivity is defined as the profit minus cost. In 
BTP, cost of traversal is not considered as an objective and there is a single vehicle available which aims to maximize the total attractiveness (profit) of the tour by selecting nodes to be visited and arcs to be travelled while having side constraints, such as route duration or cost. Profit terms appear on the objective of both problems include node and arc profit; however, their effects on one another is not studied in a bi-objective fashion.

Since, GRP includes both node and arc routing aspects, node routing and arc routing problems can be considered as special cases of GRP. Due to their closeness to the proposed problem, we study both the node (vehicle) routing problems (VRP) and the arc routing problems (ARP).

If there is a subset of nodes required to be visited with an empty required edge set, the GRP reduces to the Travelling Salesman Problem (TSP) or its multi-vehicle version VRP (Dantzig et al., 1954; Dantzig and Ramser, 1959). Travelling Purchaser Problem is defined as a generalization of TSP, in which, in contrast to TSP, nodes to be visited are not pre-specified and different selections are possible (Golden et al., 1981).

Node routing problems where the vehicle(s) performing a profit-maximizing tour with selecting customers to visit, are classified under the TSP with profits name (Feillet et al., 2005). TSPs with profits are further classified according to how they tackle the bi-objective nature of the problem, namely collected profit and travel costs. Finding a tour that maximizes the difference between the profit gained by visiting nodes and the travel cost -by subtracting the cost from the profitare categorized as Profitable Tour Problems (Dell'Amico et al., 1995; Archetti et al., 2009; Malandraki and Daskin, 1993). Other variants can be characterized based on their profit-maximizing objective while having limited time, capacity or cost constraint. Those problems are usually defined as variants of Orienteering Problem (OP) (Tsiligirides, 1984; Laporte and Martello, 1990; Kataoka and Morito, 1988; Awerbuch et al., 1998; Ramesh and Brown, 1991; Chao et al., 1996; Archetti et al., 2009; Butt and Cavalier, 1994). Another alternative for dealing with the bi-objective nature of the TSP with profits is by introducing cost minimization as an objective and profit as a constraint. This category is defined as Prize-Collecting TSP (PCTSP) by Balas (1989). In PCTSP, the aim is to minimize cost while visiting enough points to have pre-defined profit. As the profit for each vertex can be collected at most once and there is a cost associated with travel, in all node routing with profits problems, a constraint is imposed so that each customer is visited at most once.

Routing problems where customers are located at arcs on a directed network are categorized under ARPs Guan (1962); Orloff (1974). In parallel to Feillet et al. (2005), ARPs that concern with finding a profit-maximizing tour while selecting arcs to traverse can be gathered under the ARP with profits. Finding a tour that maximizes the difference between the profit gained by traversing arcs and the travel cost -by subtracting the cost from the profit- can be categorized as Profitable Arc Tour Problems (Feillet et al., 2005; Malandraki and Daskin, 1993; Aráoz et al., 2006). Where the goal is to find a maximum profit arc tour under limited time, capacity or cost consideration provides other versions of ARP with profits. Those problems are usually variants of Arc OP (Souffriau et al., 2011; Archetti et al., 2014; 2010).

The minimization of the total cost, the total distance, the number of vehicles used, and maximizing the profit or quality/customer satisfaction, and balancing the workload are the prevalent objectives in multi-objective routing problems (Jozefowiez et al., 2008). In this context, problems discussed above are implicitly multi-objective, in which objectives of profit maximization and cost minimization are present. The closest relatives of PDARP are BTP and UCGRP with profits. BTP maximizes the profit collected from visited nodes and arcs and treats cost objective as a constraint (Deitch and Ladany, 2000). The later one considers the maximization of the difference between the profit collected from visited nodes and arcs, and the cost of traversal (Archetti et al., 2017).

The proposed problem in this study, PDARP does not have required nodes or edges to be traversed, and the problem has the goals of assessing nodes and monitoring arcs. Two goals may have conflicting interests and the value of assessing an arc or node is not comparable with a single metric. Hence, the problem can be taken as a variant of bi-criteria GRP with profits. Allowing multiple node passages with the heterogeneous set of vehicles and combining two objectives in a bi-objective manner raise a new problem to the literature we refer as PDARP. As bi-objectivity of the problem is handled with the $\epsilon$-constraint method, PDARP can be considered as a variant of both TSP with profits and ARP with profits; but, contrary to both, PDARP does not have cost concerns.

\subsection{Drone applications}

As the use of motorcycles and/or drones are considered in the proposed problem, the application areas of the drone systems and the studies in the literature, in which drones are used, will be investigated.

Drone systems are primarily developed for military applications. Unmanned surveillance, inspection, and mapping areas are the leading aims for the usage of drones for the military. Recently, drones have become popular for delivery and civilian data acquisition. Large organizations like Amazon, Deutsche Post DHL, Google, the United Arab Emirates have shown interest in drone delivery (Amazon, 2016; DHL, 2014; Google, 2014; UAE, 2014). To date, there have been numbers of studies on this issue (Murray and Chu, 2015; Agatz et al., 2016; Ha et al., 2018). In Scott and Scott (2017), use of drone delivery for healthcare is discussed and mathematical models are developed to facilitate timely and efficient delivery in the noncommercial setting.

Some civilian data acquisition applications are for agriculture, forestry, archaeology, environment, emergency management and traffic monitoring (Nex and Remondino, 2014). In emergency management, drones are used for obtaining images for the impact assessment and the rescue planning. For example, in 2015 Nepal earthquake, drones assisted search and rescue teams to locate survivors (Choi-Fitzpatrick et al., 2016). Chou et al. (2010) propose an emergency drone 
application after a typhoon, while Haarbrink and Koers (2006) focus on rapid response operations such as traffic incidents. Molina et al. (2012) investigate the utilization of drones for searching the lost people. Two recent articles by Huang et al. (2017) and Giordan et al. (2017), investigates the usage of unmanned aerial vehicles in post-disaster assessment. Huang et al. (2017) claim that the drones can be used effectively in any stage of the disaster management. Furthermore, since drones do not require any on-site work, the examples of using drones for assessment of natural disasters provide an ease in gathering information in less time at a low risk (Giordan et al., 2017).

Although drone applications in the emergency management are started to be studied, they are not covered with OR perspective, rather they focus on the technicalities of such applications. Hence, as the usefulness of the drones in the disaster management is put forward, this necessitates a further study that develops effective routing policies and models to support assessment efforts.

To the best of our knowledge, there is no study that develops bi-objective routing policies and models for joint use of motorcycles and drones to support assessment efforts that focuses on both transportation network and disaster victims' needs in relief operations.

\section{Model development}

Consider a disaster-affected region as a directed, incomplete graph. Districts constitute nodes and roads constitute edges. Districts can be classified into two categories, the ones that require assessment and the ones who provide necessary forces for assessment operations, namely depots or disaster management centers. Further classification of districts can be made according to population and type of facilities they have. The ones which have facilities like hospitals, schools or have relatively high populations constitutes critical nodes. In a similar fashion, roads connecting critical nodes or the ones that blockage on it causes a significant increase in the distance travelled by disaster victims constitutes critical edges. The aim is to reach and assess critical nodes together with critical edges as soon as possible by traversing along paths that may even include debrisblocked edges. To do so, the vehicles, which are suitable for off-road conditions such as drones, motorcycles are dispatched from a depot node. Vehicles travel to reach and assess the critical nodes and the arcs in a limited time frame.

Let $G=(N, E)$ be a network where $N$ represents the nodes and E represents the edges. $A=\{(i, j) \cup(j, i): i, j \in E\}$ constitutes the arc set of the network. The node set contains the supply node $s$, and critical nodes. Also, it is worth noting that even if the arcs are directed, the parameter settings of arcs $(i, j)$ and $(j, i)$ are symmetric. If either of $(i, j)$ or $(j, i)$ is traversed, it is assumed that the condition of edge $(i, j)$ is assessed. Let $d_{i j}$ represent the distance between node $i \in N$ and node $j \in N$. We also define a parameter, $E$, for the existence of arcs. If $\operatorname{arc}(i, j)$ is in the transportation network, then $E_{i j}=1$. $E_{i j}=0$ means $\operatorname{arc}(i, j)$ does not exist.

Weights are introduced in order to present the criticality of nodes and arcs. Weight for each node in $N$ denotes importance and we assume populations will provide a good estimate for the weights. Potential population levels for the critical points like hospitals and schools are estimated by the nearest assignment of the neighbouring points' population. Node weights, $p_{i}$, are calculated with respect to the modified populations of the nodes.

The weight of arc $(i, j)$, which is denoted as $q_{i j}$, characterizes the importance of road connecting node $\mathrm{i}$ to node $\mathrm{j}$. It is calculated with respect to the criticality of the road segment and population points it connects. We define the criticality of a road segment by the total percentage change in the distance travelled by populations when the road is blocked.

Let $M$, and $D$ represent the sets of motorcycles and drones, respectively, available at the disaster management center (depot). Vehicles in respective sets $M$ and $D$, are considered to be identical and cardinality of these sets are $|M|$ and $|D|$. Let $V$ represent the set of all vehicles available at the disaster management center (depot). Note that set $V$ consists of vehicles in $M$ and $D$ in an ordered fashion where first $n m$ vehicles are motorcycles. As previously discussed, candidate vehicles are taken as off-road motorcycles and/or drones. Average velocity $v$ is given accordingly. The output of the model will be $n m+n d$ tours each of which starts their tour and returns to the depot within a predetermined time bound $T$.

If the vehicle is in the set of motorcycles, $M$, assessment of arcs and nodes is only possible by traversing them. If the vehicle is in the set of drones, $D$, as drones have angular point of view; flying over arc $(i, j)$ may result with also assessment of nodes $m, n$ and arcs $(i, m),(i, n),(j, m),(j, n),(m, n)$. Parameters $a_{i j}^{l}$ and $b_{i j}^{l m}$ are introduced to denote node and arc monitoring capabilities of drones over each arc. If drone flying over arc $(i, j)$ can monitor node $l$, then $a_{i j}^{l}=1$. $a_{i j}^{l}=0$ means drone cannot assess node $l$ through flying over arc $(i, j)$. Similarly, if drone flying over arc $(i, j)$ can make assessment on arc $(l, m)$, then $b_{i j}^{l m}=1$. $b_{i j}^{l m}=0$ means drone cannot assess node $l$ through flying over arc $(i, j)$. Assessment capabilities of drones, $a_{i j}^{l}$ and $b_{i j}^{l m}$, are calculated with respect to the distances from nodes $l$ and $m$ to arc $(i, j)$. If the distances from nodes $l$ and $m$ to arc $(i, j)$ are below some threshold, it is assumed that $a_{i j}^{l}$ and $b_{i j}^{l m}$ take value 1 . For example, as in Fig. 1 , consider a drone flying over arc (1,2), the shaded area around the traversed arc marks the assessment region of the drone. The nodes and the arcs that lie entirely in the shaded region are considered to be assessed by flying over arc $(1,2)$.

In the context of general routing with profit, Archetti et al. (2017), prove that every directed arc in the graph can be traversed at most twice by vehicle k. We will make use of this result in our model. Similar to the Fig. 1, a basic node-arc diagram of the proposed model can be provided (See Fig. 2.). Consider a disaster network as depicted in the Fig. 2a. With 1 drone and 1 motorcycle, depending on the distance and the weight values, it is possible to observe routes as given in the Fig. 2b. Since there is a time-bound for vehicles, some nodes cannot be visited. In Fig. 2b, grey-coloured arcs define motorcycle route while black-coloured arcs make up the route of a drone. Starting from the depot node, drone flies to the 


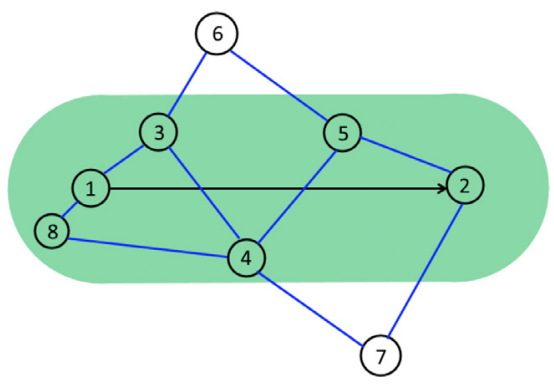

Fig. 1. Illustrative example of angular point of view of a drone.

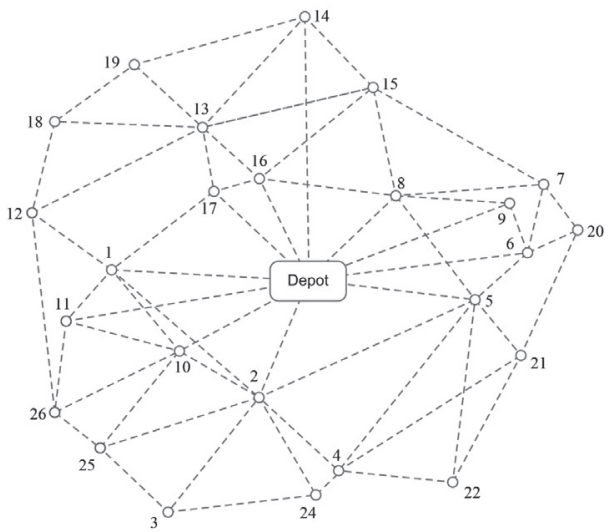

(a) An example disaster network diagram

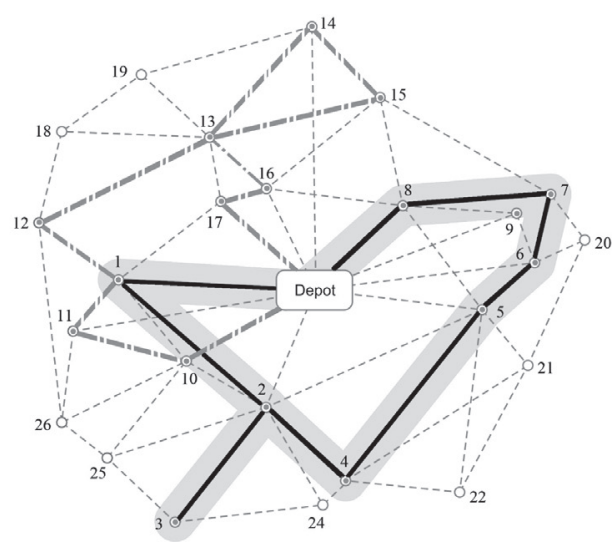

(b) An example routes of a proposed model for 1 drone and 1 motorcycle on a given network

Fig. 2. An example node-arc diagram and possible routes of proposed model.

following nodes in sequence, 1, 2, 3, 2, 4, 5, 6, 7, 8 and motorcycle visit 10,11, 12, 13, 14, 15, 13, 16 and 17, then they return to the depot node.

As in Fig. 1, the shaded region around the black coloured arc marks the assessment region of the drone. The nodes and the arcs that lie entirely in the shaded region are being assessed by flying over a given route. Assessed network elements that lie in the shaded region are the nodes 9 and 10 , and the arcs $(1,10),(2,10)$ and $(8,9)$. However, only the nodes and arcs that lie in the motorcycle route, coloured grey, are considered to be assessed. There are two non-depot nodes in the figure which are visited multiple times, node 2 and 13. Also, node 10 which lies in the shaded region around the drone route is visited along the motorcycle route. Although its assessment can be conducted with the visit of the motorcycle, assessment of the arcs emerging from it that lie in the shaded region is only possible with the drone (assessment of the arcs $(1,10)$ and $(2,10)$ ). In the figure, nodes with dots represent the nodes being assessed by either of the vehicles. It is important to note that the twice traversal of an arc is not depicted in the figure to avoid complications arising from the superposition of routes.

\subsection{Mathematical model}

In this section, we introduce a bi-objective mixed-integer linear programming model which determines the paths of the vehicles with the $\epsilon$-constraint method. Three-step solution approach is constructed for the problem. At first, only the weights of the assessed arcs are maximized within a period of time and this problem is called arc profit PDARP. Then, in parallel, only the weights of the monitored nodes are maximized in node profit PDARP while respecting the time bounds of vehicles. As a final step, to address both issues simultaneously, arc profit is maximized in the objective while collected node weights is assured to be at least equal to predetermined $(\epsilon)$ level.

Before presenting the optimization model for the post-disaster assessment routing problem, we provide the nomenclature. 
Sets:

Note that $V$ is an ordered set of $M$ and $D$.

Depot node is denoted by $s \in N$.

\section{Parameters:}

\begin{tabular}{ll}
\hline$N$ & Set of all nodes. \\
$A$ & Set of all arcs. \\
$M$ & Set of motorcycles. \\
$D$ & Set of drones. \\
$V$ & Set of vehicles. $V=M \cup D$. \\
\hline
\end{tabular}

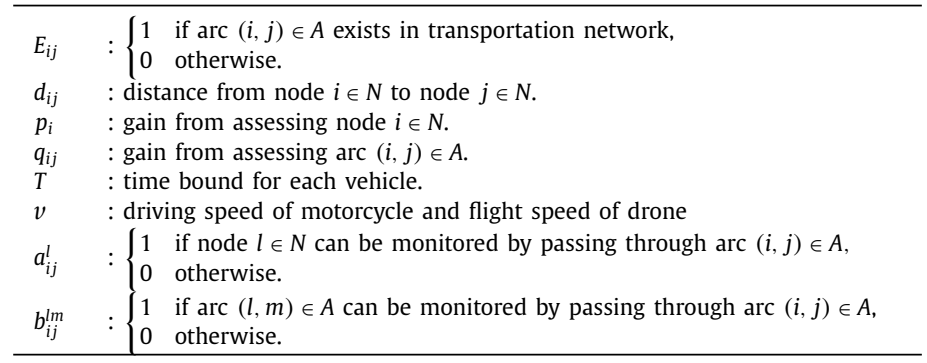

The decisions to be made can be represented by the following sets of variables:

\section{Decision Variables:}

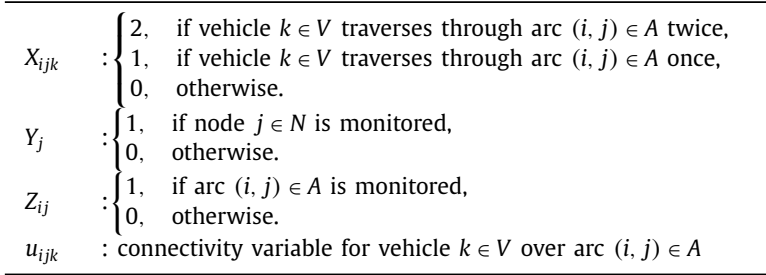

The following mixed integer linear program for PDARP can now be proposed:

maximize $f 1, f 2$

subject to

$f 1=\sum_{\substack{i<j \\(i, j) \in A}} q_{i j} \cdot Z_{i j}$

$f 2=\sum_{j \in N} p_{j} \cdot Y_{j}$

$X_{i j k} \leq 2 \cdot E_{i j} \quad \forall(i, j) \in A, \forall k \in V$

$Z_{i j} \leq 1 \cdot E_{i j} \quad \forall(i, j) \in A$

$\sum_{i \in N} X_{i j k}-\sum_{i \in N} X_{j i k}=0 \quad \forall j \in N, \forall k \in V$

$Y_{j} \leq \sum_{i \in N}\left(\sum_{k \in M} X_{i j k}+\sum_{l \in N} \sum_{k \in D} a_{i l}^{j} \cdot X_{i l k}\right) \quad \forall j \in N$

$Y_{j} \geq \frac{1}{2} \cdot X_{i j k} \quad \forall(i, j) \in A, \forall k \in M$

$Y_{j} \geq a_{i l}^{j} \cdot \frac{1}{2} \cdot X_{i l k} \quad \forall(i, l) \in A, \forall j \in N, \forall k \in D$

$Z_{i j} \leq \sum_{k \in M}\left(X_{i j k}+X_{j i k}\right)+\sum_{k \in D} \sum_{(l, m) \in A}\left(b_{l m}^{i j} \cdot X_{l m k}\right) \quad \forall(i, j),(j, i) \in A$ 


$$
\begin{aligned}
& Z_{i j} \geq \frac{1}{2 \cdot 2} \cdot\left(X_{i j k}+X_{j i k}\right) \quad \forall(i, j),(j, i) \in A \forall k \in M \\
& Z_{i j} \geq \frac{1}{2} \cdot\left(b_{l m}^{i j} \cdot X_{l m k}\right) \quad \forall(i, j),(l, m) \in A, \forall k \in D \\
& \sum_{i \in N} X_{i s k}=1 \quad \forall k \in V \\
& \sum_{j \in N} X_{s j k}=1 \quad \forall k \in V \\
& \sum_{(i, j) \in A} d_{i j} \cdot X_{i j k} \leq v \cdot T \quad \forall k \in V \\
& \sum_{j \in N}\left(u_{i j k}-u_{j i k}\right)-\sum_{j \in N} d_{i j} \cdot X_{i j k}=0 \quad \forall i \in N \backslash\{s\}, \forall k \in V \\
& u_{s j k}=d_{s j} \cdot X_{s j k} \quad \forall j \in N \backslash\{s\}, \forall k \in V \\
& u_{i s k} \leq v \cdot T \cdot X_{i s k} \quad \forall i \in N \backslash\{s\}, \forall k \in V \\
& u_{i j k} \leq\left(v \cdot T-d_{j s}\right) \cdot X_{i j k} \quad \forall(i, j) \in A, j \neq s, \forall k \in V \\
& u_{i j k} \leq \max \left\{v \cdot T-d_{j s}, 0\right\} \quad \forall(i, j) \in A, j \neq s, \forall k \in V \\
& u_{i j k} \geq\left(d_{s i}+d_{i j}\right) \cdot \frac{1}{2} \cdot X_{i j k} \quad \forall(i, j) \in A, i \neq s, \forall k \in V \\
& X_{i j k} \in\{0,1,2\}, \quad \forall(i, j) \in A, \forall k \in V \\
& Z_{i j} \in\{0,1\}, \quad \forall(i, j) \in A \\
& Y_{j} \in\{0,1\}, \quad \forall j \in N
\end{aligned}
$$

The objective function (0) maximizes the total importance of arcs and nodes assessed. We remind here that although we are working on a directed graph, assessments are made through monitoring either direction.

As $X_{i j k}, Z_{i j}$ are defined for each node pair, constraints (3) and (4) are imposed to guarantee that each arc traversed/assessed exists in the ground transportation network. Constraint (5) specifies the flow balance conditions for vehicle $k$. Constraints (6)-(8) monitor the assessment of node $j$ by any of the vehicles. Constraints (9)-(11) check if arc ( $i, j)$ is monitored by any vehicles in either direction. Constraints (12) and (13) ensure all vehicles leave the depot once and return once. Total distance bound is given by the constraint (14). Constraint (15) ensures the connectivity of the tour for each vehicle $k$. Constraint (16) calculates the distance travelled by vehicle $k$, leaving the depot. By constraints (17)-(19), an upper bound on non-depot entering connectivity variable is imposed. To explain further, constraint (17) bounds the ones entering the depot by the total travel distance limit. Constraint (18) bounds the non-depot entering ones by considering the travel distance limit and the distance which has to be travelled to return the depot. Constraint (19) imposes a positive distance bound on the non-depot entering connectivity variables. By constraint (20), we ensure that connectivity variables take a positive value when a vehicle traverses that particular network element. Therefore, disconnected tours are eliminated via constraints (15)-(20). Note that when $X_{i j k}=0$, they force $u_{i j k}$ to be 0 ; while they force $u_{i j k}$ to be between $\left(d_{s i}+d_{i j}\right) \cdot \frac{1}{2} \cdot X_{i j k}$ and $\left(v \cdot T-d_{j s}\right)$ when $X_{i j k}>0$ for $j \neq s$. In this way, multiple visits to nodes are allowed while avoiding disconnected sub-tours. Constraints (21)-(23) are domain constraints.

In the objective function (0), we have two terms to maximize which are defined by (1) and (2). To tackle the biobjectivity, we use $\epsilon$-constraint method. It is critical to note that as the problem is a mixed integer program, resulting Pareto 
Table 1

$\epsilon$-constrained mathematical models.

\begin{tabular}{ll}
\hline Arc Profit PDARP & Node Profit PDARP \\
\hline $\begin{array}{l}\text { maximize } f 1 \\
\text { subject to }\end{array}$ & $\begin{array}{c}\text { maximize } \\
\text { subject to }\end{array}$ \\
$(1)-(23)$ & $(1)-(23)$ \\
$f 2 \geq \epsilon_{2} \quad\left(\epsilon_{2}=v+\epsilon\right)(24)$ & $f 1 \geq \epsilon_{1} \quad\left(\epsilon_{1}=\rho\right)(25)$ \\
\hline
\end{tabular}

frontier may have Pareto efficient solutions which cannot be found using weighted-sum scalarization technique. Additionally, weighted-sum scalarization with fixed weights would return only one of the Pareto-efficient points. Since assessment of the node or arc have distinct implications on the disaster management operations, and their importance is calculated using different metrics, we prefer to utilize a bi-objective methodology. The following additional parameters are defined for the $\epsilon$-constraint method.

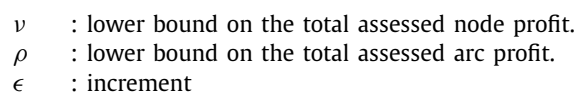

For the Arc Profit PDARP, objective $f 1$ is taken as the objective and $f 2$ is considered as a constraint. For the Node Profit PDARP, objective function $f 1$ is replaced by objective $f 2$ and $\epsilon$-constraint (24) is replaced with the constraint (25). The mathematical models are given in Table 1.

Initially, Arc Profit PDARP is solved without the $\epsilon$-constraint and $\epsilon_{1}$ is set to its optimal objective function value $f_{1}^{*}$. Then, Node Profit PDARP is solved in order to find the best objective function value having the same $f_{1}^{*}$ value. Lets say $f_{2}^{*}$ is the objective function value. The resulting objective values for $f_{1}^{*}$ and $f_{2}^{*}$ are recorded as one of the Pareto efficient solutions. After this, $\epsilon_{2}$ is equated to $f_{2}^{*}+\epsilon$ in order to find the next Pareto efficient solution by solving Arc Profit PDARP again and following the similar procedure as the initial step. These algorithmic steps are repeated until the infeasibility in solving Arc Profit PDARP occurs.

In order to justify the utilization of $\epsilon$-constraint method, we show that optimal solutions of Table 1 problems are at least weakly efficient. We first need to provide some definitions.

Let $\Gamma$ be the domain defined by the constraints (1)-(23) and the respective $\epsilon$-constraint, (24) or (25).

A feasible solution $\bar{X} \in \Gamma$ is called efficient or Pareto optimal, if there is no $X \in \Gamma$ such that $f(x) \geq f(\bar{X})$. If $\bar{X}$ is efficient, $f(\bar{X})$ is a non-dominated point.

A feasible solution $\bar{X} \in \Gamma$ is called weakly efficient (weakly Pareto optimal) if there is no $X \in \Gamma$ such that $f(X)>f(\bar{X})$, i.e. $f_{j}(X)>f_{j}(\bar{X})$ for all $j=1,2$. The point $f(\bar{X})$ is then called weakly non-dominated.

Proposition 1. Let $\bar{X}$ be an optimal solution of one of the problems in Table 1 for some $j \in\{1,2\}$, then $\bar{X}$ is weakly efficient.

Proof. Assume $\bar{X}$ is not weakly efficient. Then $\exists k \in\{1,2\}$ and $X \in \Gamma$ such that $f_{k}(X)>f_{k}(\bar{X})$. Let us say $f_{j}(X)>f_{j}(\bar{X})$ for $k \neq j$, the solution $X$ is feasible for one of the problems in Table 1 for some $j \in\{1,2\}$. This contradicts to $\bar{X}$ being an optimal solution of one of the problems in Table 1 for some $j \in\{1,2\}$.

Corollary 1. Let $X \in \Gamma$ be a solution of one of the problems in Table 1 with an optimality gap, then Pareto optimality of the solution $X$ cannot be asserted. Thus, $f(x)$ is called Pareto approximate.

Remark 1. In one of the iterations, if optimal solution to the problems in Table 1 can be found, then optimal solution is weakly optimal Pareto point. If it cannot be found at that iteration, the best solution found so far is called approximate Pareto point.

Remark 2. If there is at least one approximate Pareto point in an instance, then the resulting Pareto front is called approximate.

During our preliminary computational analysis, we observe that PDARP is a computationally challenging problem. Warmstarting Arc Profit PDARP is considered as a method to reduce the computation time. A slightly different optimization problem can be utilized to obtain an initial point for the current problem for the warm-start procedure. Therefore, we propose a version of the Arc Profit PDARP to find feasible starting point. To do so, we redefine $X_{i j k}$ so that second pass is not allowed.

Updated Decision Variable:

$X_{i j k}^{\prime}: \begin{cases}1, & \text { if vehicle } k \text { traverses through arc }(i, j) \in A, \\ 0, & \text { otherwise. }\end{cases}$

The following mixed integer linear program for Arc Profit 1-PDARP can now be proposed:

maximize $f 1$

subject to

(1) - (2), (4) - (6), (9), (12) - (19), (22) - (24) 


$$
\begin{array}{ll}
X_{i j k}^{\prime} \leq E_{i j} & \forall(i, j) \in A, \forall k \in V \\
Y_{j} \geq X_{i j k}^{\prime} & \forall(i, j) \in A, \forall k \in M \\
Y_{j} \geq a_{i l}^{j} \cdot X_{i l k}^{\prime} & \forall(i, l) \in A, \forall j \in N, \forall k \in D \\
Z_{i j} \geq \frac{1}{2} \cdot\left(X_{i j k}^{\prime}+X_{j i k}^{\prime}\right) & \forall(i, j),(j, i) \in A, \forall k \in M \\
Z_{i j} \geq b_{l m}^{i j} \cdot X_{l m k}^{\prime} & \forall(i, j),(l, m) \in A, \forall k \in D \\
u_{i j k} \geq\left(d_{s i}+d_{i j}\right) \cdot X_{i j k}^{\prime} & \forall(i, j) \in A, i \neq s, \forall k \in V \\
X_{i j k}^{\prime} \in\{0,1\} & \forall(i, j) \in A, \forall k \in V
\end{array}
$$

Proposition 2. A feasible solution to Arc Profit 1-PDARP is also feasible to the Arc Profit PDARP.

Proof. Take a feasible solution $\bar{X}$ of Arc Profit 1-PDARP. The constraints (1), (2), (4)-(6), (9), (12)-(19),(22), (23), (24) are automatically satisfied as they are the same for both problems. The feasible solution $\bar{X}$ to restricted problem satisfies $\bar{X} \subset X$ where $\bar{X}=\left\{\bar{X}_{i j k}: \bar{X}_{i j k} \in\{0,1\} \forall(i, j) \in A, \forall k \in V\right\}$ and $X=\left\{X_{i j k}: X_{i j k} \in\{0,1,2\} \forall(i, j) \in A, \forall k \in V\right\}$. To prove $\bar{X}$ 's feasibility for the original problem, we need to check whether $\bar{X}$ satisfies remaining constraints of the Arc Profit PDARP.

- As $E$ is a non-negative matrix, $E_{i j} \leq 2 \cdot E_{i j} \quad \forall(i, j) \in A$. For feasible $\bar{X}, \bar{X}_{i j k} \leq E_{i j} \quad \forall(i, j) \in A$ is satisfied by the constraint (3a). So, the following is satisfied $\bar{X}_{i j k} \leq 2 \cdot E_{i j} \quad \forall(i, j) \in A$. Hence, $\bar{X}$ does not violate the constraint (3).

- The constraint (7a), $Y_{j} \geq \bar{X}_{i j k} \quad \forall(i, j) \in A, \forall k \in M$, is satisfied by feasible $\bar{X}$ and $\bar{X}_{i j k} \geq \frac{1}{2} \cdot\left(\bar{X}_{i j k}\right) \quad \forall(i, j) \in A, \forall k \in M$ for $\bar{X}$. Hence, the constraint (7), $Y_{j} \geq \frac{1}{2} \cdot X_{i j k} \quad \forall(i, j) \in A, \forall k \in M$ holds.

- For any $\bar{X}, \bar{X}_{i l k} \geq \frac{1}{2} \cdot \bar{X}_{i l k} \quad \forall(i, l) \in A, \forall k \in D$ is satisfied. $Y_{j} \geq a_{i l}^{j} \cdot \bar{X}_{i l k} \quad \forall(i, l) \in A, \forall k \in D$ for feasible $\bar{X}$ by the constraint (8a). Considering two inequalities together, $Y_{j} \geq a_{i l}^{j} \cdot \bar{X}_{i l k} \geq a_{i l}^{j} \cdot \frac{1}{2} \cdot \bar{X}_{i l k} \quad \forall(i, l) \in A, \forall k \in D$. So, the constraint (8) is not violated by feasible solution $\bar{X}$.

- Take constraint (10a), $Z_{i j} \geq \frac{1}{2} \cdot\left(\bar{X}_{i j k}+\bar{X}_{j i k}\right) \quad \forall(i, j),(j, i) \in A, \forall k \in M$. This constraint is satisfied with feasible solution $\bar{X}$. And for any $\bar{X}, \frac{1}{2} \cdot\left(\bar{X}_{i j k}+\bar{X}_{j i k}\right) \geq \frac{1}{2 \cdot 2} \cdot\left(\bar{X}_{i j k}+\bar{X}_{j i k}\right) \quad \forall(i, j),(j, i) \in A, \forall k \in M$. Hence, feasible solution $\bar{X}$ will also satisfy the constraint (10) which is $Z_{i j} \geq \frac{1}{2 \cdot 2} \cdot\left(X_{i j k}+X_{j i k}\right) \quad \forall(i, j),(j, i) \in A, \forall k \in M$.

- For feasible solution $\bar{X}$, constraint (11a) holds. That is, $Z_{i j} \geq b_{l m}^{i j} \cdot \bar{X}_{l m k} \quad \forall(i, j),(l, m) \in A, \forall k \in D$. For feasible solution $\bar{X}$ and non-negative matrix $b$, the following inequality holds: $b_{l m}^{i j} \cdot \bar{X}_{l m k} \geq \frac{1}{2} \cdot b_{l m}^{i j} \cdot \bar{X}_{l m k} \quad \forall(i, j),(l, m) \in A, \forall k \in D$. So, the following is satisfied $Z_{i j} \geq \frac{1}{2} \cdot b_{l m}^{i j} \cdot \bar{X}_{l m k} \quad \forall(i, j),(l, m) \in A, \forall k \in D$. Thus, $\bar{X}$ does not violate the constraint (11).

- The constraint (20a), $u_{i j k} \geq\left(d_{s i}+d_{i j}\right) \cdot \bar{X}_{i j k} \quad \forall(i, j) \in A, \forall k \in V$, is satisfied by feasible $\bar{X}$ and $\left(d_{s i}+d_{i j}\right) \cdot \bar{X}_{i j k} \geq\left(d_{s i}+d_{i j}\right)$. $\frac{1}{2} \cdot \bar{X}_{i j k} \quad \forall(i, j) \in A, \forall k \in V$ for $\bar{X}$. So, the constraint $(20), u_{i j k} \geq\left(d_{s i}+d_{i j}\right) \cdot \frac{1}{2} \cdot X_{i j k} \quad \forall(i, j) \in A, \forall k \in V$ holds for $\bar{X}$.

Remark 3. Feasible region of the arc profit 1-PDARP is tighter than the arc profit PDARP.

Hence, the feasible solution $\bar{X}$ found for the model that restricts traversal of arcs by at most once provide a feasible solution to Arc Profit PDARP. Thus, we first formulated the single pass version of the problem and warm-started the PDARP with the paths we generated with the single-pass one. In this way, branch-and-bounding process is speeded up by providing a good starting point and eliminating the possibly inferior solutions.

\section{Heuristic solution methodologies for PDARP}

Experiments we conducted with the mathematical model has shown that as the bound on node criticality increase, it is harder to reach the optimal solution in the course of a reasonable time frame. It may take hours to find the Pareto optimal solution for some instances. However, due to the problem characteristics, immediate decisions are required. Therefore, we decided to develop a heuristic solution methodology, which can find a set of good Pareto solutions within the scope of solution quality and time trade-off.

For that purpose, we developed a fast constructive heuristic solution method which we refer to as Base Route Heuristic $(\mathrm{BRH})$, based on some maximum profit definitions. To find a set of good Pareto solutions, we also applied improvement heuristic methodologies. 


\subsection{Construction}

BRH constructs paths that start from the depot node and return in an allowed time frame. The algorithm uses the shortest path network between each critical node calculated over the existing network via Dijkstra's algorithm. For each vehicle, the first node is selected as the one with the most profit among the feasible ones. At each step, one additional node is inserted into each path among the most profitable ones without violating time bound. Then, until the time limit violation, the same steps are applied. When no shortest path satisfying time condition is found, the last inserted path segment is removed and a path to the depot is added.

It is assumed that vehicles assess the nodes and edges of this constructed path (motorcycle, drone) or assesses the nodes and edges nearby (drone). Since after a node or edge is assessed, it cannot be assessed again. Hence, the assessment operation is not conducted if it is traversed by motorcycle or it is in point of view of a drone again. While calculating profits to be collected, this assumption is taken into consideration. Only the new assessments are considered in profit calculations.

A node to be inserted in the path is determined based on one of the 4 profit definitions. The profit can be a value added by traversing arc/node, or a ratio of value added by traversing arc/node per distance. The value added by traversing a network element corresponds to a change in either objective function value. Heuristic solution methods rely on those 4 construction methods and apply the consecutive improvement operations on the corresponding constructed paths. An illustrative example of the construction algorithm can be found at Appendix A.

\subsection{Random improvement}

In a broad sense, random improvement heuristic searches for a new solution by generating random solutions from the current solution. During the search, it records each result found that does not violate the time constraint. After some number of iterations, dominated solutions are eliminated from the pool of records. Then the algorithm returns all the non-dominated paths in the solution pool.

Our improvement heuristic consists of five random improvement algorithms: swap, insertion, reversion, add and removeadd. At each iteration, a new random solution is generated by randomly calling one of the five improvement algorithms. As we have multiple vehicles, there are multiple routes to consider in each improvement heuristics.

In each improvement algorithm applications, a route to apply the algorithm is determined randomly in multi-vehicle problem instances. For each algorithm, Let depot $\rightarrow i 1 \rightarrow i 2 \rightarrow i 3 \rightarrow \ldots . \rightarrow j 1 \rightarrow j 2 \rightarrow j 3 \rightarrow$ depot be the given route. If the operation specific conditions are not met, the operation rerun till success or till reaching the maximum number of trials.

\subsubsection{Swap}

The swap algorithm randomly chooses two non-depot nodes on the route and exchanges their positions. Let us say algorithm chooses $i 2$ and $j 2$ nodes from the route. If arcs $(i 1, j 2),(j 2, i 3),(j 1, i 2)$, and $(i 2, j 3)$ exist in the transportation network, the swap operation is successfully done. Then, the new route is depot $\rightarrow i 1 \rightarrow j 2 \rightarrow i 3 \rightarrow \ldots \ldots \rightarrow j 1 \rightarrow i 2 \rightarrow j 3 \rightarrow$ depot.

\subsubsection{Insertion}

Two locations on a given route are randomly chosen, then, a node in the left location is moved to another location in the right by shifting subsequent elements of the paths to left. Let us say algorithm chooses locations of $i 2$ and $j 2$ on the route and $i 2$ is subjected to move. If $i 1 \neq i 3, j 2 \neq i 2, i 2 \neq j 3$ and arcs $(i 1, i 3),(j 2, i 2),(i 2, j 3)$ exist in the transportation network, insertion can be successfully performed. Then, the new route is depot $\rightarrow i 1 \rightarrow i 3 \rightarrow \ldots \ldots \rightarrow j 1 \rightarrow j 2 \rightarrow i 2 \rightarrow j 3 \rightarrow$ depot.

\subsubsection{Reversion}

The algorithm randomly chooses two non-depot nodes on the route and reverses the path segment in between. Let us say algorithm chooses $i 2$ and $j 2$ nodes from the route. If arcs $(i 1, j 2)$, and $(i 2, j 3)$ exist in the transportation network, reversion operation is successfully done. Then algorithm returns the new route, depot $\rightarrow i 1 \rightarrow j 2 \rightarrow j 1 \rightarrow \ldots . i 3 \rightarrow i 2 \rightarrow$ $j 3 \rightarrow$ depot.

\subsubsection{Add}

In the algorithm, a non-depot node from $N$ and a location to add the new node on a given route are randomly chosen. Let us say algorithm chooses a non-depot node $k 1$ and a location of $i 2$ on the route. There are two conditions that lead to success. First, if $k 1 \neq i 1, k 1 \neq i 2$, and arcs $(i 1, k 1),(k 1, i 2)$ exist in the transportation network, add operation can be successfully performed. Then, the new route is depot $\rightarrow i 1 \rightarrow k 1 \rightarrow i 2 \rightarrow i 3 \rightarrow \ldots+j 1 \rightarrow j 2 \rightarrow j 3 \rightarrow$ depot. The other condition for successful operation is having $j 1 \neq i 2$, and arc $(j 1, i 2)$ 's existence in the transportation network. Then, the resulting route is depot $\rightarrow i 1 \rightarrow i 2 \rightarrow k 1 \rightarrow i 2 \rightarrow i 3 \rightarrow \ldots . j 1 \rightarrow j 2 \rightarrow j 3 \rightarrow$ depot.

\subsubsection{Remove-Add}

In the remove-add algorithm, a non-depot node from $N$ and a location to remove and add the new node on a given route are randomly chosen. Let us say algorithm chooses a non-depot node $k 1$ and a location of $i 2$ on the route. If $k 1 \neq i 1, k 1 \neq i 3$, 
and $\operatorname{arcs}(i 1, k 1),(k 1, i 3)$ exist in the transportation network, the remove-add operation can be successfully performed. Then, the resulting route is depot $\rightarrow i 1 \rightarrow j 1 \rightarrow i 3 \rightarrow \ldots . \rightarrow j 1 \rightarrow j 2 \rightarrow j 3 \rightarrow$ depot.

A detailed pseudo-code combining the construction, BRH, and random improvement heuristic is given in Algorithm 1 .

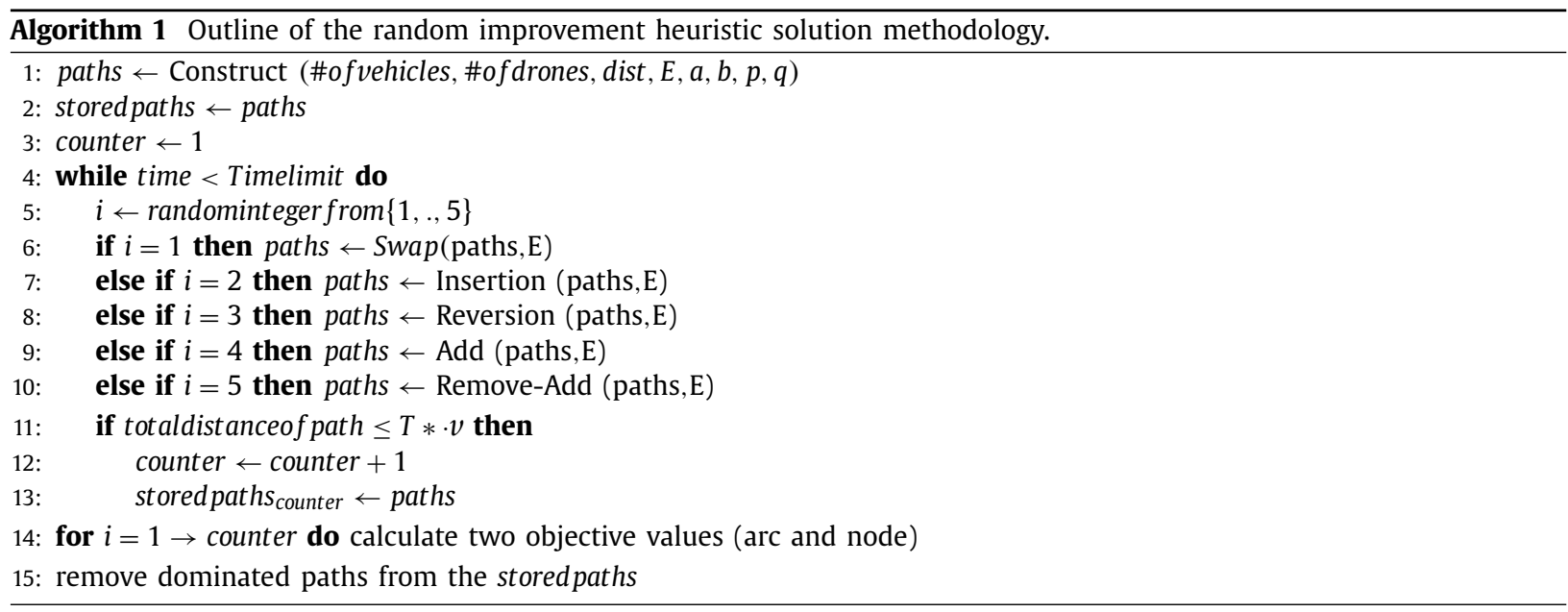

The integral part of the algorithm lies in the construction of the initial paths. Algorithm 2 performs this task. Improvement

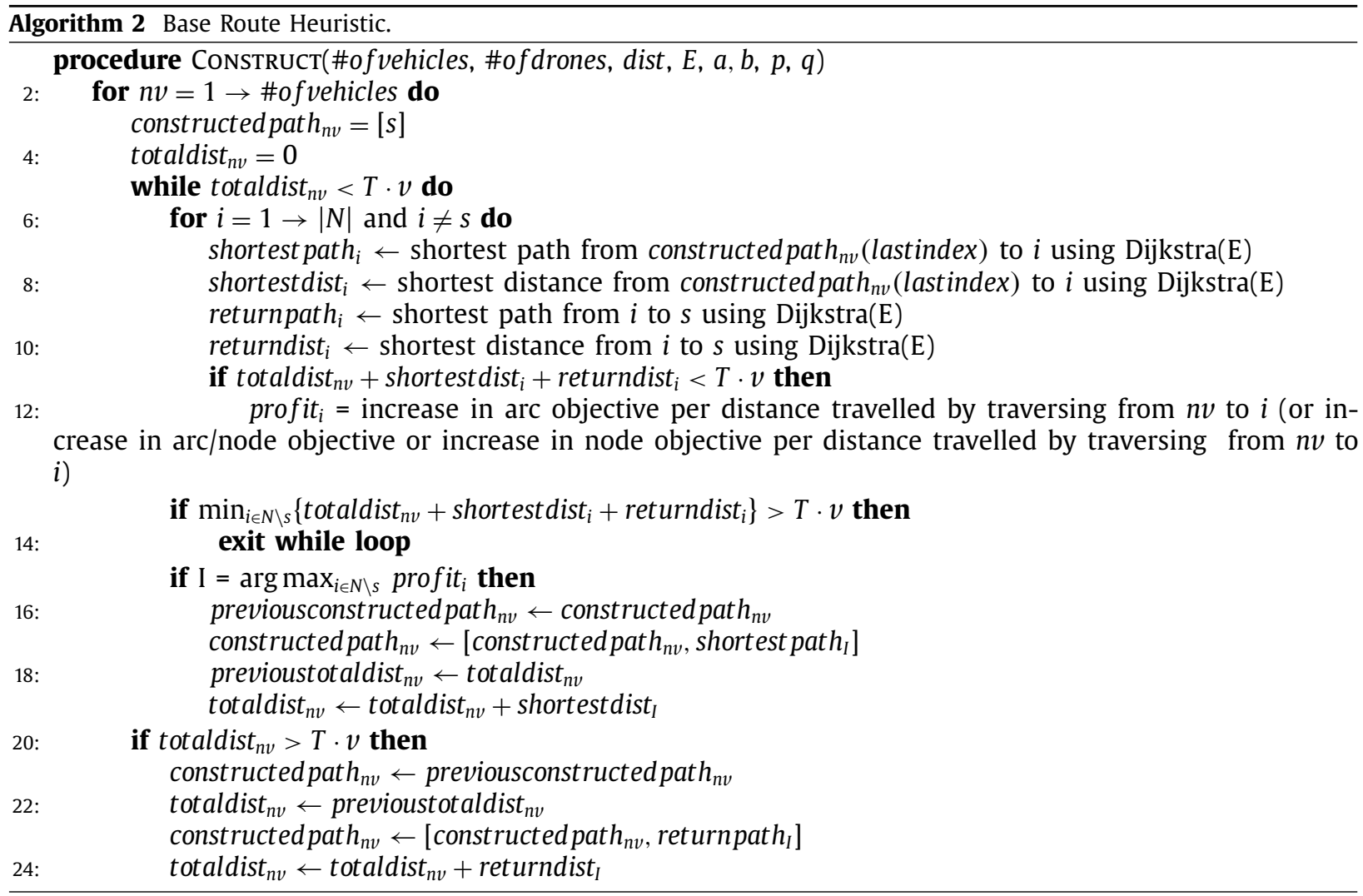

heuristics are visualized in the Fig. 3.

\subsection{Purposive improvement}

Similar to the random improvement heuristic, the purposive improvement heuristic searches for new solutions by generating random solutions from the current solution. Instead of randomly moving between solutions, purposive improvement 


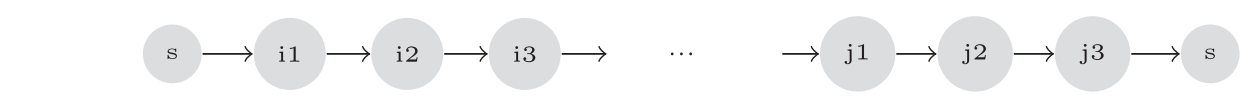

(a) Input of improvement algorithms

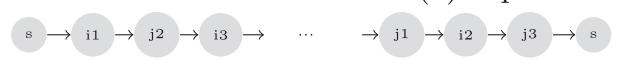

(b) Output of Swap: the locations of nodes $i 2$ and $j 2$
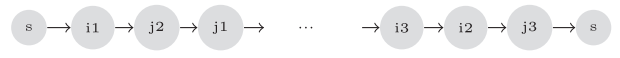

(d) Output of Reversion: the locations of nodes $i 2$ and $j 2$
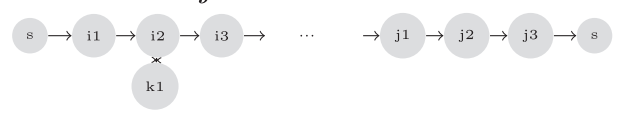

(f) Output of Add (alternative 2): the location of node $i 2$ and node $k 1$

\section{$\mathrm{s} \rightarrow \mathrm{i} 1 \rightarrow \mathrm{i} 3 \rightarrow$}

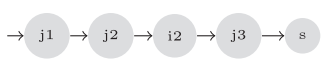

(c) Output of Insertion: the locations of nodes $i 2$ and $j 2$

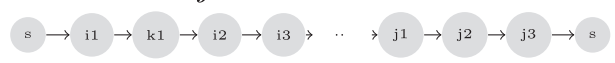

(e) Output of Add (alternative 1): the location of nodes $i 2$ and node $k 1$

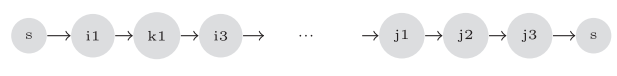

(g) Output of Remove-Add: the location of node $i 2$ and node $k 1$

Fig. 3. Illustrative example of the improvement algorithms.

Table 2

Features of the data set.

\begin{tabular}{ll}
\hline & Kartal municipality \\
\hline Number of Nodes & 45 \\
Symmetric distance matrix & Yes \\
Ground transportation network & Yes \\
Depot node (node number) & 16 \\
Number of schools (node numbers) & $3(14,21,22)$ \\
Number of hospitals (node numbers) & $4(26,33,41,43)$ \\
\hline
\end{tabular}

seeks the best improvement on both objectives under a random number of iterations using all of the improvement algorithms. During the search, procedure records the two best results in terms of arc, and node assessment objectives found that does not violate the time constraint. After a predetermined number of iterations, dominated solutions are eliminated from the pool of solutions. Then the algorithm returns all the non-dominated routes in the solution pool.

The proposed purposive improvement heuristic consists of the same five improvement algorithms: swap, insertion, reversion, add and remove-add as in the improvement heuristic. At each iteration, five new solutions are generated by calling the five improvement algorithms. Note here that there are multiple routes to consider in each improvement heuristics due to the multiple number of vehicles.

Recall the five improvement algorithms, this time the randomness in the improvement algorithms is eliminated, meaning, the improvement algorithms are called with the same parameters. Note here that there are multiple routes to consider in each improvement heuristics due to the multiple number of vehicles. The first parameter is a route to apply the algorithm in multi-vehicle problem instances. The others are the two locations $i 2, j 2$ from the route, and a node, $k 1 \in N$. If the operation specific conditions are not met for all of the algorithms, the same operation is repeated with new parameters till success.

A detailed pseudo-code combining the construction, and purposive improvement heuristics is given in Algorithm 3 . Algorithm 2 performs an integral part of the Algorithm 3 which is the initial path's construction.

\section{Computational analysis}

\subsection{Data}

To measure the effectiveness of the developed mathematical model, and the heuristic solution methodology, we used a data set from Turkey based on Istanbul's Kartal district (Kilci et al., 2018). Kartal is specified as the 11th most crowded district among the 39 districts of Istanbul and has nearly 425,000 inhabitants.

There are 20 sub-districts in Kartal and the population of each is assumed to be concentrated in its center. Moreover, there are 25 points of interests (POI) which are determined as emergency rallying points. These POIs include school yards, mall parking lots and some other appropriate points. The locations of 45 nodes are presented in Fig. 4. Sub-districts together with POIs are taken as population points as POIs have the possibility of being densely populated during the disaster. There are 7 POIs containing schools and hospitals. Those are illustrated with yellow squares and green stars, respectively, while red dots are used for other nodes. The Marmara Region Disaster Center of the Turkish Red Crescent, which is located in Kartal, is considered as a candidate depot for disaster relief operations and represented by a red triangle in Fig. 4. Features of the data set are summarized in Table 2 . 

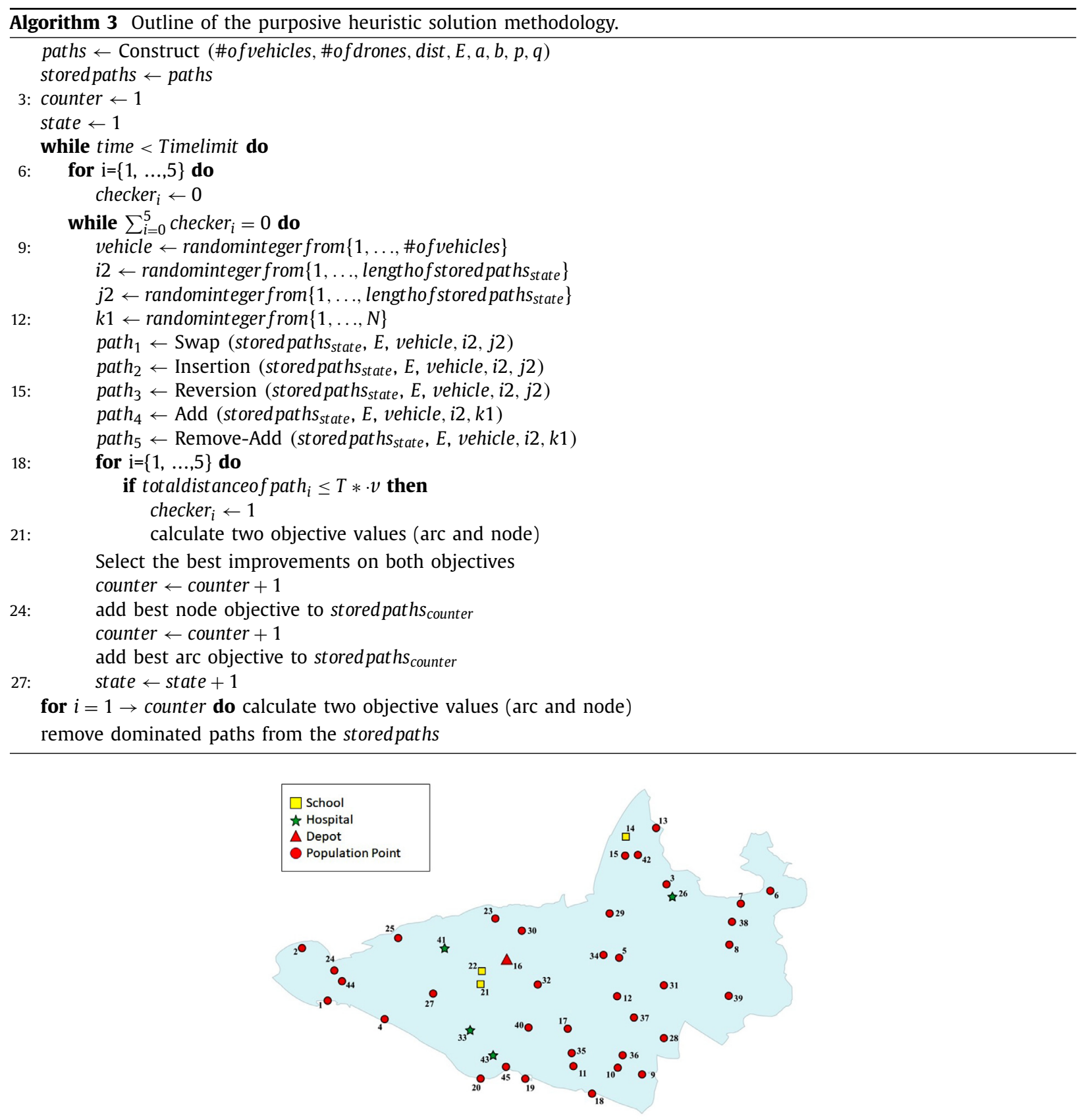

Fig. 4. The location of depot and critical nodes in Kartal municipality (Kilci et al., 2015).

In order to determine the critical elements of a network, certain weights are assigned to the nodes and arcs. For nodes, the weights are determined based on the number of people living in a district. To give a relatively higher importance to the points like hospitals and schools, potential population levels are determined. To do so, the populations of the districts are aggregated and assigned to each nearest hospital and school. Node weights $\left(p_{i}\right)$ are calculated with respect to the resulted aggregated populations and reduced to the $[0,1]$ interval.

On the other hand, the importance of roads is determined based on the population of the points that it connects and criticality of the road. If blockage on the road causes a significant increase in the distance travelled by disaster victims and the weights of the nodes connected by that road are high, then the importance of the arc increases along with the weight value assigned to it. Hence, the weight assigned to the arc $(i, j)$ is directly proportional to the shortest path distance change from node $i$ to $j$ when $(i, j)$ is blocked and the sum of the populations at $i$ and $j$ and inversely proportional to the complete network distance. 
Table 3

Test parameters of the model.

\begin{tabular}{llll}
\hline Driving/Flight Speed & $40 \mathrm{~km} / \mathrm{hr}$ \\
Epsilon $(\epsilon)$ & $0.001, \quad 0.01, \quad 0.1$ \\
Number of Drones & $1, \quad 2$ & \\
Number of Motorcycles & $1, \quad 2,3$ & \\
Time bound & $2 \mathrm{hr}$ & \\
\hline
\end{tabular}

Table 4

Weights of nodes and arcs for solutions with 1 motorcycle, 0 drone.

\begin{tabular}{llll}
\hline & Solution $a$ & Solution $b$ & Solution $c$ \\
\hline Total weights of Assessed Nodes & 5.826 & 6.220 & 6.015 \\
Total weights of Assessed Arcs & 5.737 & 4.995 & 5.383 \\
\hline
\end{tabular}

The number of vehicles available for assessment operations considered to be at most 2 drones and 3 motorcycles. The reason is that after a disaster, resources are scarce and available vehicles may be allocated to other response operations. Due to its endurance and camera specifications, Aeromapper Talon model of Aeromao Inc. is considered as a candidate vehicle (Aeromao, 2014). It has a cruise speed of $50 \mathrm{~km} / \mathrm{h}$, considering take-off and landing, average drone speed assumed is about $40 \mathrm{~km} / \mathrm{h}$. Additionally, for the motorcycles, the vehicle speed is considered to be again about $40 \mathrm{~km} / \mathrm{h}$, which is suitable for off-road capable motorcycles. As the first hours after a disaster is critical for response operations, time-window for vehicles is set to 2 hours in order to make the assessment in a short period of time. This 2 hours time limit is also in line with the endurance of the Aeromapper Talon drone. The range is another property to be considered in utilizing drones; Aeromapper Talon has a range of $30+$ which underlines its usefulness in assessment. Under this parameter setting, it is not required to include a constraint regarding range properties.

Maximum flight altitude for a drone is restricted in most countries by $120 \mathrm{~m}$. Chosen drone, Aeromapper Talon, has Sony A6000 model camera mounted on it (Aeromao, 2014). Sony A6000 camera can have a focal length ranging from $16 \mathrm{~mm}$ to $50 \mathrm{~mm}$. As focal length gets smaller, the field of view increases, so the focal length setting is taken as $16 \mathrm{~mm}$. With the focal length of $16 \mathrm{~mm}$ and a flight altitude of $120 \mathrm{~m}$, it is possible to monitor all the points within a circle of $75-80 \mathrm{~m}$ radius. In this study, we take this radius as $75 \mathrm{~m}$. Considering a densely populated city such as Istanbul, using drones for assessment purposes promises evaluation of nearby points and roads while passing through a particular path. To that end, we introduced parameters for node and arc monitoring capabilities of drones over each arc. If a point $m$ is within $75 \mathrm{~m}$ distance to any point on arc $(i, j)$, its assessment can be done while flying over arc $(i, j)$. Similarly, each arc connecting the points lie within $75 \mathrm{~m}$ of distance to any point on arc $(i, j)$ can be assessed by traversing through arc $(i, j)$ by drone.

Since mathematical model accounts for $\epsilon$-value, it is determined by the smallest weight difference between population points and it is taken as 0.001. Larger $\epsilon$-values, 0.1 and 0.01 , are also used in order to compare the resulting frontiers in terms of quality, how well they approximate the Pareto front, and the computational requirement of the overall procedure. In Table 3, the parameters of the model are summarized.

\subsection{Computation experiments}

All computations are performed on a 4xAMD Opteron Interlagos $2.6 \mathrm{GHz}$ processor and 96 GB RAM computer with Linux operating system. The MIP model is solved using CPLEX 12.6. The heuristic algorithms were coded in Matlab R2015b on the Intel Core i7-4702MQ $2.2 \mathrm{GHz}$ processor with $8 \mathrm{~GB}$ RAM computer with Windows 10 operating system.

To illustrate the computational results of the PDARP, routes of a sample instance are given in Fig. 5 which consists of 1 motorcycle and no drones, under $\epsilon=0.001$. In the Fig. 5a, Pareto efficient solutions obtained by solving the bi-objective model iteratively is illustrated. Solution $a$ corresponds to the arc profit PDARP where $v$ and $\epsilon$ value are set to 0 . Solution $b$ corresponds to the node profit PDARP in which $\epsilon$ takes the highest possible value so that the model acts as if the node profit is maximized without any violation or it can be obtained by solving node profit PDARP model. Solution $c$ is one of the non-dominated solutions in the Pareto frontier. The routes of a motorcycle in these three solutions can be found in Fig. 5b, c, and d respectively.

In solution $a$, it is observed that certain nodes like node 43 are visited multiple times to traverse critical arcs as much as possible in 2 hours. Node 43 is a hospital; therefore, it is logical to traverse that node multiple times and route on the arcs connected to it as they have higher weights. On the other hand, in solution $b$, it is recognized that each node is visited at most once to be able to increase the overall node profits. Therefore, even if the multiple passages are allowed, the model performs as if it is a classical routing problem. Finally, in solution $c$, the number of nodes visited and overall node profit collected are higher than solution $a$ while multiple passages through some nodes are observed contrary to solution $b$. This indicates that the bi-objective solution clearly demonstrates the characteristics of both single-objective solution approaches. The total weights of the visited nodes and traversed arcs can be found in Table 4. As it is seen, best overall arc weight and node weight are obtained in solution $a$ and $b$, respectively. Solution $c$ seems to compensate for the drawbacks of both solutions and provides a somewhat balanced solution in terms of both objective values. 


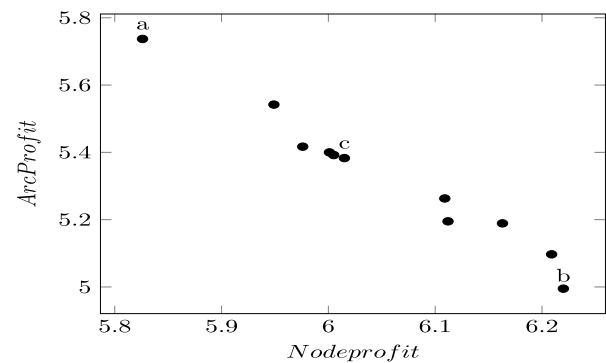

(a) Pareto Chart when the number of motorcycles is 1 , the number of drones is 0

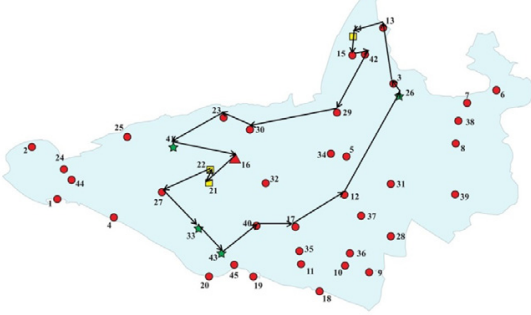

(c) Node profit PDARP (Solution $b$ )

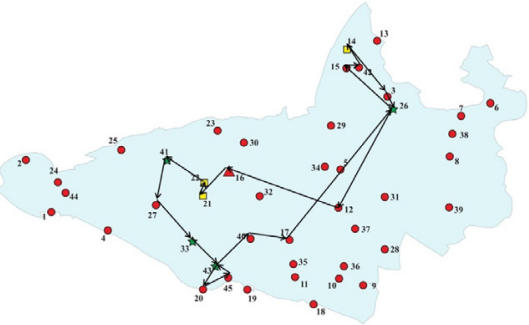

(b) Arc Profit PDARP (Solution $a$ )

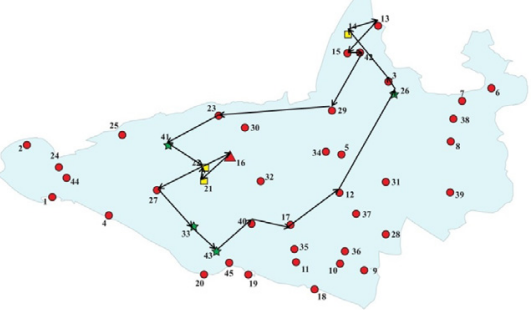

(d) An Example Non-dominated Pareto Solution (Solution $c$ )

Fig. 5. Comparison of solutions with 1 motorcycle.

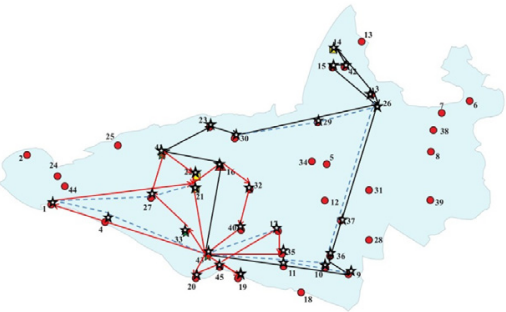

(a) Arc profit PDARP with 2 drones

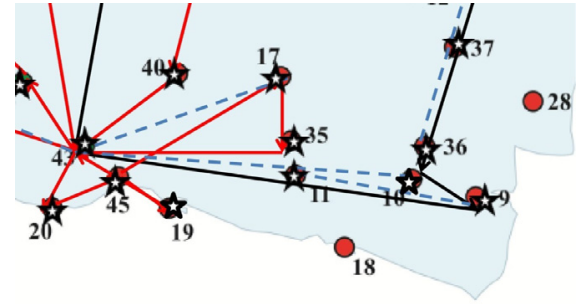

(b) Zoomed view of arc profit PDARP with 2 drones

Fig. 6. Assessing nearby nodes and arcs by using drones for assessment purposes.

Using drones for assessment purposes ensure assessment of the nearby points and roads. This characteristic can be observed in the Fig. 6 where the routes of 2 drones and no motorcycles instance are given. In the figure, the solid lines (red, black) indicate the arcs lie in the flight paths of drones, while the dashed lines reflect the assessed arcs that do not lie in the flight paths. For example, taking the figure into consideration, arcs $(43,17),(10,11)$ are not in any of the drones' paths, but they are monitored through flying over arcs $(9,43),(17,45)$. Similarly, roads $(9,11),(11,43)$, and $(37,36)$ are assessed by flying over the roads parallel to them.

It is important to recall Corollary 1 at this point. Thus; when a solution is obtained with a gap during the $\epsilon$-constraint procedure, resulting Pareto front is called as approximate. In Table 5, model performances are summarized for $\epsilon=0.001$. It can be seen that in 8 instances out of 11, we could not find exact Pareto fronts for PDARP while allocating 2 hours to arc profit 1-PDARP and 4 hours to arc profit PDARP as a solution time for finding each Pareto solution. In 6 of the instances, the mathematical model was able to find some of the exact Pareto optimal solutions but not all of them. Average solution time required for an exact/approximate Pareto solution is over 3 hours in 7 of the instances and is more than 4.5 hours in 6 of them. When the number of drones is 1 or 0 , the total number of Pareto solutions tends to be much higher compared to the 2 drone cases. Additionally, having 3 or more vehicles at hand result with approximate Pareto solutions where allowed solution time is mostly consumed.

As in the most of the cases, having exact Pareto solutions is computationally difficult, for this reason, we utilized larger $\epsilon$-values and developed our heuristic methodologies that quickly reach good, feasible Pareto solutions. Table 6 depicts the performance results of the model under different $\epsilon$ values. In each instance, the number of Pareto solutions, CPU require- 
Table 5

Model performances of Kartal instances under $\epsilon=0.001$.

\begin{tabular}{|c|c|c|c|c|c|}
\hline Instance $(|D|,|M|)$ & $\begin{array}{l}\text { Number of Total } \\
\text { Pareto Solutions }\end{array}$ & $\begin{array}{l}\text { Number of Exact } \\
\text { Pareto Solutions }\end{array}$ & $\begin{array}{l}\text { Number of } \\
\text { Approximate Pareto } \\
\text { Solutions }\end{array}$ & $\begin{array}{l}\text { Total Solution Time } \\
\text { per Pareto solution } \\
\text { (hours) }\end{array}$ & Average GAP (\%) \\
\hline$(0,1)$ & 11 & 11 & - & 0.05 & 0.00 \\
\hline$(0,2)$ & 70 & 47 & 23 & 3.07 & 1.33 \\
\hline$(0,3)$ & 25 & - & 25 & 5.84 & 3.97 \\
\hline$(1,0)$ & 19 & 18 & 1 & 1.66 & 0.06 \\
\hline$(1,1)$ & 22 & 22 & - & 0.94 & 0.00 \\
\hline$(1,2)$ & 10 & - & 10 & 5.98 & 1.76 \\
\hline$(1,3)$ & 4 & - & 4 & 6.00 & 1.50 \\
\hline$(2,0)$ & 8 & 8 & - & 0.58 & 0.00 \\
\hline$(2,1)$ & 6 & 2 & 4 & 4.83 & 0.70 \\
\hline$(2,2)$ & 4 & - & 4 & 6.00 & 1.49 \\
\hline$(2,3)$ & 1 & - & 1 & 6.00 & 1.10 \\
\hline
\end{tabular}

Table 6

Model performances under $\epsilon$ values: $0.001,0.01,0.1$.

\begin{tabular}{|c|c|c|c|c|c|c|}
\hline Instance $(|D|,|M|)$ & $\epsilon$ values & $\begin{array}{l}\text { Number of Pareto } \\
\text { Solutions }\end{array}$ & $\begin{array}{l}\text { Number of Exact } \\
\text { Pareto Solutions }\end{array}$ & Total CPU (hours) & $\begin{array}{l}\text { Total CPU per Pareto } \\
\text { solution (hours) }\end{array}$ & Average GAP (\%) \\
\hline \multirow[t]{3}{*}{$(0,1)$} & 0.001 & 11 & 11 & 0.46 & 0.06 & 0.00 \\
\hline & 0.01 & 9 & 9 & 0.43 & 0.05 & 0.00 \\
\hline & 0.1 & 4 & 4 & 0.29 & 0.07 & 0.00 \\
\hline \multirow[t]{3}{*}{$(0,2)$} & 0.001 & 70 & 47 & 214.91 & 3.07 & 1.33 \\
\hline & 0.01 & 55 & 47 & 115.99 & 2.11 & 0.40 \\
\hline & 0.1 & 13 & 11 & 27.73 & 2.13 & 0.20 \\
\hline \multirow[t]{3}{*}{$(0,3)$} & 0.001 & 25 & - & 146.03 & 5.84 & 3.97 \\
\hline & 0.01 & 21 & - & 125.29 & 5.97 & 3.12 \\
\hline & 0.1 & 10 & - & 59.29 & 5.93 & 2.51 \\
\hline \multirow[t]{3}{*}{$(1,0)$} & 0.001 & 19 & 18 & 31.51 & 1.66 & 0.06 \\
\hline & 0.01 & 16 & 16 & 23.66 & 1.48 & 0.00 \\
\hline & 0.1 & 8 & 8 & 12.09 & 1.51 & 0.00 \\
\hline \multirow[t]{3}{*}{$(1,1)$} & 0.001 & 22 & 22 & 20.67 & 0.94 & 0.00 \\
\hline & 0.01 & 16 & 16 & 7.40 & 0.46 & 0.00 \\
\hline & 0.1 & 6 & 6 & 3.06 & 0.51 & 0.00 \\
\hline \multirow[t]{3}{*}{$(1,2)$} & 0.001 & 10 & - & 60.00 & 6.00 & 1.76 \\
\hline & 0.01 & 10 & - & 40.00 & 6.00 & 1.18 \\
\hline & 0.1 & 4 & - & 24.00 & 6.00 & 1.08 \\
\hline \multirow[t]{3}{*}{$(1,3)$} & 0.001 & 4 & - & 24.00 & 6.00 & 1.50 \\
\hline & 0.01 & 3 & - & 18.00 & 6.00 & 1.59 \\
\hline & 0.1 & 2 & - & 12.00 & 6.00 & 1.54 \\
\hline \multirow[t]{3}{*}{$(2,0)$} & 0.001 & 8 & 8 & 4.69 & 0.58 & 0.00 \\
\hline & 0.01 & 6 & 6 & 3.13 & 0.52 & 0.00 \\
\hline & 0.1 & 3 & 3 & 0.86 & 0.29 & 0.00 \\
\hline \multirow[t]{3}{*}{$(2,1)$} & 0.001 & 6 & 2 & 28.98 & 4.83 & 0.70 \\
\hline & 0.01 & 5 & 2 & 22.67 & 4.53 & 0.66 \\
\hline & 0.1 & 3 & 2 & 8.72 & 2.90 & 0.37 \\
\hline \multirow[t]{3}{*}{$(2,2)$} & 0.001 & 4 & - & 24.00 & 6.00 & 1.49 \\
\hline & 0.01 & 4 & - & 24.00 & 6.00 & 1.16 \\
\hline & 0.1 & 2 & - & 12.00 & 6.00 & 1.13 \\
\hline \multirow[t]{3}{*}{$(2,3)$} & 0.001 & 1 & - & 6.00 & 6.00 & 1.10 \\
\hline & 0.01 & 1 & - & 6.00 & 6.00 & 1.10 \\
\hline & 0.1 & 1 & - & 6.00 & 6.00 & 1.10 \\
\hline
\end{tabular}

ments, and solution gap are reported. As expected, with larger $\epsilon$ values, the Pareto front is approximated in a shorter period of time at the expense of obtaining less Pareto points. By using $\epsilon=0.01,19 \%$ of the Pareto points are lost while the computational time is decreased by $31 \%$. Taking $\epsilon$ as 0.1 enables us to spend $70 \%$ less time on computation; however, in turn, we lose $69 \%$ of the Pareto points. Beside total computational time benefits, larger $\epsilon$ values turn out to be effective in decreasing the average solution gap and total CPU time per each Pareto solution. As it can be observed in Table 7, utilizing larger epsilon values provides a great benefit in decreasing computational time requirements at the expense of finding a worse off Pareto front approximation. Heuristic approaches' performance in reducing the computational effort requirement stands out, as the heuristics provide a CPU time reduction up to 99.5\%. Although heuristic approaches show a significant performance in terms of solution time, their performances' should be further investigated.

The performance of the larger $\epsilon$-values and the heuristic algorithms should be evaluated and it is essential to use quantitative performance metrics to evaluate the quality of the approximate Pareto front. To this end, three popular performance 
Table 7

Decrease (\%) in CPU time requirements with the mathematical models under $\epsilon=0.01, \epsilon=0.1$ and heuristics with random and purposive improvement in CPU time requirement.

\begin{tabular}{llllllllllll}
\hline \multicolumn{1}{c}{ Instance $(|D|,|M|)$} \\
\cline { 2 - 12 } & $(0,1)$ & $(0,2)$ & $(0,3)$ & $(1,0)$ & $(1,1)$ & $(1,2)$ & $(1,3)$ & $(2,0)$ & $(2,1)$ & $(2,2)$ & $(2,3)$ \\
\hline$\epsilon=0.01$ & 4.4 & 46.0 & 14.2 & 24.9 & 64.2 & 33.3 & 25.0 & 33.3 & 21.8 & 0.0 & 0.0 \\
$\epsilon=0.1$ & 36.9 & 87.1 & 59.4 & 61.6 & 85.4 & 60.0 & 50.0 & 81.7 & 69.9 & 50.0 & 0.0 \\
Random & - & 99.5 & 99.3 & 96.8 & 95.2 & 98.3 & 95.8 & 78.7 & 96.5 & 95.8 & 83.3 \\
Purposive & - & 99.5 & 99.3 & 96.8 & 95.2 & 98.3 & 95.8 & 78.7 & 96.5 & 95.8 & 83.3 \\
\hline
\end{tabular}

measures are used, which are spacing metric (S)(Schott, 1995), maximum spread metric (MS)(Hyun et al., 1998), and set coverage (SC) (Zitzler and Thiele, 1998). These three metrics can be used with approximate Pareto solutions set.

Spacing (S): To measure the spread uniformity of the solutions set this measure can be used. The definition of this metric is as follows:

$$
\begin{aligned}
& S=\sqrt{\frac{1}{n-1} \cdot \sum_{i=1}^{n}\left(d_{i}-\bar{d}\right)^{2}} \\
& d_{i}=\min _{j} \sum_{k=1}^{m}\left|O_{k}^{i}-O_{k}^{j}\right| \quad i, j=1,2, \ldots, n
\end{aligned}
$$

where $n$ is the number of non-dominated solutions in the solution pool, $m$ is the number of objectives, $O_{k}^{i}$ is the $k^{\text {th }}$ objective value of a solution $i$ and $\bar{d}$ is the mean value of all $d_{i}$ (Schott, 1995). When $\mathrm{S}$ is close to zero, it means all the points spread uniformly. That is, the smaller $S$ value is better in terms of diversity.

Maximum Spread (MS): This metric measures the maximum extension covered by the non-dominated solution set, i.e., the maximum Euclidean distance between a solution and other non-dominated solutions in that set (Hyun et al., 1998). Mathematically, it is defined by the following equation:

$$
M S=\sqrt{\sum_{i=1}^{n} \max _{\substack{j=\{1, \ldots, n\} \\ j \neq i}}\left(\left\|O^{i}-O^{j}\right\|\right)}
$$

where $\left\|O^{i}-O^{j}\right\|$ is the Euclidian distance between objective values of $O^{i}$ and $O^{j}$ in the solution set of heuristic or mathematical model. In this equation, $n$ indicates the number of non-dominated solutions in the set.

Set Coverage (SC): This metric is used to compare two sets of non-dominated solutions found by two different methods (Zitzler and Thiele, 1998). Let method 1 and method 2 be sets of solutions found by two different methods. SC(method1, method2) is the ratio of points that are dominated by or equal to at least one point in method1. It is expressed by the following equation:

$$
S C(\text { method } 1, \text { method } 2)=\frac{\left|\left\{O^{i} \in \operatorname{method} 2 \mid \exists O^{j} \in \operatorname{method} 1: O^{i} \leq 0^{j}\right\}\right|}{|\operatorname{method} 2|}
$$

If $S C(\operatorname{method} 1, \operatorname{method} 2)=1$, all points in method 2 are dominated by or equal to some points in method 1 , while the $S C(\operatorname{method} 1, \operatorname{method} 2)=0$ implies the opposite. As there are intersections between these sets, both $S C(\operatorname{method} 1, \operatorname{method} 2)$ and $S C$ (method2, method1) should be considered. We can say that second method is better than the first in terms of accuracy if and only if $S C(\operatorname{method} 1, \operatorname{method} 2)=0$ and $S C(\operatorname{method} 2, \operatorname{method} 1)=1$ (Zitzler and Thiele, 1998).

Average Maximum Spread (AMS): As MS is heavily affected by the number of non-dominated solutions found by the method, Average Maximum Spread measure is proposed which is equal to Maximum Spread per non-dominated solutions found. Larger the AMS value is, the better the solution set is because higher AMS value indicates the solutions are more spread and the set is able to cover further edges of the Pareto front.

Both heuristics and mathematical models' results and performance measure values are summarized in Table $8,9,10$. The solution times of the random and purposive heuristics are around $1 \mathrm{~h}$ for each instance.

In Table 8, the performances of the mathematical models under different epsilon values are compared in terms of spacing, maximum spread, and average maximum spread. Spacing metric measures the deviation distance from the average solution, which is higher in the model with $\epsilon=0.1$ than the models with other epsilon values. The reason for this can be the following: the number of points returned by the model with $\epsilon=0.001$ is larger and those points are located close to each other, thus the deviation (S) is lower. Hence, the model with $\epsilon=0.001$ is performing better by having lower deviation. Another diversity metric Maximum Spread is also higher in the model with $\epsilon=0.001$, which can be explained with the larger number of Pareto points. Additionally, Average Maximum Spread metric attains its' highest values with the $\epsilon=0.1$. This result can be attributed to the approximation of Pareto front with a fewer number of points. 
Table 8

Performance analysis of mathematical models with $\epsilon=0.001, \epsilon=0.01$ and $\epsilon=0.1$ using performance metrics.

\begin{tabular}{|c|c|c|c|c|c|c|c|c|c|c|}
\hline \multicolumn{2}{|c|}{ Instance } & \multicolumn{3}{|l|}{ Spacing } & \multicolumn{3}{|c|}{ Maximum Spread } & \multicolumn{3}{|c|}{ Average Maximum Spread } \\
\hline$|D|$ & $|M|$ & $\begin{array}{l}\text { Model } \\
(\epsilon=0.001)\end{array}$ & $\begin{array}{l}\text { Model } \\
(\epsilon=0.01)\end{array}$ & $\begin{array}{l}\text { Model } \\
(\epsilon=0.1)\end{array}$ & $\begin{array}{l}\text { Model } \\
(\epsilon=0.001)\end{array}$ & $\begin{array}{l}\text { Model } \\
(\epsilon=0.01)\end{array}$ & $\begin{array}{l}\text { Model } \\
(\epsilon=0.1)\end{array}$ & $\begin{array}{l}\text { Model } \\
(\epsilon=0.001)\end{array}$ & $\begin{array}{l}\text { Model } \\
(\epsilon=0.01)\end{array}$ & $\begin{array}{l}\text { Model } \\
(\epsilon=0.1)\end{array}$ \\
\hline 0 & 1 & 0.008 & 0.008 & 0.001 & 2.585 & 2.370 & 1.600 & 0.235 & 0.263 & 0.400 \\
\hline 0 & 2 & 0.008 & 0.006 & 0.025 & 14.362 & 12.780 & 5.613 & 0.205 & 0.232 & 0.432 \\
\hline 0 & 3 & 0.003 & 0.002 & 0.005 & 6.205 & 5.702 & 3.999 & 0.248 & 0.272 & 0.400 \\
\hline 1 & 0 & 0.007 & 0.007 & 0.237 & 7.264 & 6.625 & 4.708 & 0.382 & 0.414 & 0.588 \\
\hline 1 & 1 & 0.005 & 0.027 & 0.089 & 5.227 & 4.501 & 2.899 & 0.238 & 0.281 & 0.483 \\
\hline 1 & 2 & 0.005 & 0.005 & 0.012 & 2.394 & 2.391 & 1.327 & 0.239 & 0.239 & 0.332 \\
\hline 1 & 3 & 0.003 & 0.000 & 0.000 & 0.766 & 0.685 & 0.482 & 0.192 & 0.228 & 0.241 \\
\hline 2 & 0 & 0.001 & 0.022 & 0.020 & 2.645 & 2.243 & 1.348 & 0.331 & 0.374 & 0.449 \\
\hline 2 & 1 & 0.004 & 0.001 & 0.005 & 1.700 & 1.592 & 1.062 & 0.283 & 0.318 & 0.354 \\
\hline 2 & 2 & 0.002 & 0.001 & 0.000 & 0.755 & 0.737 & 0.583 & 0.189 & 0.184 & 0.291 \\
\hline 2 & 3 & NAN & NAN & NAN & 0.000 & 0.000 & 0.000 & 0.000 & 0.000 & 0.000 \\
\hline
\end{tabular}

Table 9

Performance analysis of mathematical models and heuristics with random and purposive improvement using set coverage metric.

\begin{tabular}{|c|c|c|c|c|c|c|c|c|c|c|c|}
\hline & \multicolumn{11}{|c|}{ Instance $(|D|,|M|)$} \\
\hline & $(0,1)$ & $(0,2)$ & $(0,3)$ & $(1,0)$ & $(1,1)$ & $(1,2)$ & $(1,3)$ & $(2,0)$ & $(2,1)$ & $(2,2)$ & $(2,3)$ \\
\hline$\epsilon=0.1$ & 0.444 & 0.218 & 0.429 & 0.500 & 0.375 & 0.400 & 0.667 & 0.500 & 0.600 & 0.500 & 1.000 \\
\hline$\epsilon=0.01$ & 1.000 & 0.923 & 0.700 & 1.000 & 1.000 & 1.000 & 1.000 & 1.000 & 1.000 & 1.000 & 1.000 \\
\hline$\epsilon=0.1$ & 0.364 & 0.186 & 0.480 & 0.421 & 0.273 & 0.364 & 0.500 & 0.375 & 0.500 & 0.500 & 1.000 \\
\hline$\epsilon=0.001$ & 1.000 & 1.000 & 0.900 & 1.000 & 1.000 & 1.000 & 1.000 & 1.000 & 1.000 & 0.500 & 1.000 \\
\hline$\epsilon=0.01$ & 0.818 & 0.757 & 0.680 & 0.842 & 0.727 & 1.000 & 0.750 & 0.750 & 0.833 & 1.000 & 1.000 \\
\hline$\epsilon=0.001$ & 1.000 & 0.964 & 0.762 & 1.000 & 1.000 & 0.800 & 1.000 & 1.000 & 1.000 & 0.500 & 1.000 \\
\hline random & 0.000 & 0.000 & 0.000 & 0.000 & 0.000 & 0.000 & 0.000 & 0.000 & 0.000 & 0.000 & 0.000 \\
\hline $\operatorname{model}(\epsilon=0.001)$ & 1.000 & 1.000 & 1.000 & 0.800 & 1.000 & 1.000 & 1.000 & 0.875 & 1.000 & 1.000 & 1.000 \\
\hline purposive & 0.000 & 0.000 & 0.000 & 0.526 & 0.000 & 0.000 & 0.000 & 0.000 & 0.000 & 0.000 & 0.000 \\
\hline $\operatorname{model}(\epsilon=0.001)$ & 1.000 & 1.000 & 1.000 & 0.583 & 1.000 & 1.000 & 1.000 & 0.722 & 1.000 & 1.000 & 1.000 \\
\hline random & 1.000 & 1.000 & 1.000 & 0.167 & 0.235 & 0.875 & 0.250 & 0.333 & 1.000 & 0.700 & 1.000 \\
\hline purposive & 0.000 & 0.000 & 0.000 & 0.600 & 0.714 & 0.000 & 0.125 & 0.375 & 0.000 & 0.000 & 0.000 \\
\hline
\end{tabular}

Table 10

Performance analysis of mathematical model with $\epsilon=0.001$ and heuristics with random and purposive improvement using performance metrics.

\begin{tabular}{|c|c|c|c|c|c|c|c|c|c|c|}
\hline \multicolumn{2}{|c|}{ Instance } & \multicolumn{3}{|c|}{ Spacing } & \multicolumn{3}{|c|}{ Maximum Spread } & \multicolumn{3}{|c|}{ Average Maximum Spread } \\
\hline$|D|$ & $|M|$ & Model & $\begin{array}{l}\text { Random } \\
\text { Heuristic }\end{array}$ & $\begin{array}{l}\text { Purposive } \\
\text { Heuristic }\end{array}$ & Model & $\begin{array}{l}\text { Random } \\
\text { Heuristic }\end{array}$ & $\begin{array}{l}\text { Purposive } \\
\text { Heuristic }\end{array}$ & Model & $\begin{array}{l}\text { Random } \\
\text { Heuristic }\end{array}$ & $\begin{array}{l}\text { Purposive } \\
\text { Heuristic }\end{array}$ \\
\hline 0 & 1 & 0.008 & 0.579 & 0.413 & 2.585 & 2.802 & 2.435 & 0.235 & 0.700 & 0.812 \\
\hline 0 & 2 & 0.008 & 0.021 & 1.038 & 14.362 & 3.749 & 3.461 & 0.205 & 0.536 & 0.865 \\
\hline 0 & 3 & 0.003 & 0.015 & 0.000 & 6.205 & 8.622 & 3.015 & 0.248 & 0.375 & 1.507 \\
\hline 1 & 0 & 0.007 & 0.009 & 0.029 & 7.264 & 1.803 & 5.150 & 0.382 & 0.361 & 0.429 \\
\hline 1 & 1 & 0.005 & 0.011 & 0.006 & 5.227 & 3.509 & 8.543 & 0.238 & 0.501 & 0.503 \\
\hline 1 & 2 & 0.005 & 0.067 & 0.051 & 2.394 & 4.334 & 6.284 & 0.239 & 0.619 & 0.785 \\
\hline 1 & 3 & 0.003 & 0.006 & 0.003 & 0.766 & 3.035 & 5.932 & 0.192 & 0.379 & 1.483 \\
\hline 2 & 0 & 0.001 & 0.241 & 0.113 & 2.645 & 4.530 & 8.788 & 0.331 & 0.566 & 0.488 \\
\hline 2 & 1 & 0.004 & 0.012 & 0.031 & 1.700 & 2.160 & 7.090 & 0.283 & 0.432 & 0.788 \\
\hline 2 & 2 & 0.002 & 0.032 & 0.077 & 0.755 & 1.991 & 6.031 & 0.189 & 0.498 & 0.603 \\
\hline 2 & 3 & - & 0.012 & 0.006 & 0.000 & 2.100 & 1.300 & 0.000 & 0.525 & 0.325 \\
\hline
\end{tabular}

Set Coverage (SC) provides a comparative analysis and it measures the ratio of (weakly) domination by the other set. SC metrics show how better an algorithm is than one another. The closer the SC value to 1, the better the solution is. If points of the method $A$ dominate all points of the method $B$, then by the definition $S C(A, B)$ equals to 1 . However, this information alone is not enough to compare $A$ and $B$. Both $S C(A, B)$ and $S C(B, A)$ values should be considered to determine which method is better, due to intersections between the two sets.

Now, the performance of the model under different $\epsilon$ values, and the heuristic methodologies, provided in Table 9, will be discussed and compared using SC metric. If the performance of the mathematical models with different $\epsilon$ values is compared, on average $51 \%$ of the points obtained with $\epsilon=0.1$ dominates the points obtained with $\epsilon=0.01$ whereas $97 \%$ of the points obtained with $\epsilon=0.1$ are dominated by $\epsilon=0.01$. Similarly, the points obtained with $\epsilon=0.001$ dominate $95 \%$ of $\epsilon=0.1$ and $91 \%$ of $\epsilon=0.01$. In turn, $45 \%$ and $83 \%$ of the points obtained with $\epsilon=0.1$ and $\epsilon=0.01$ are dominated by 
Table 11

Improvement heuristics comparison with respect to their contribution to arc and node objectives.

\begin{tabular}{llll}
\hline Instance $(|D|,|M|)$ & Heuristics & Improvement \% in Arc Objective & Improvement \% in Node Objective \\
\hline$(0,1)$ & Random & $\mathbf{2 0}$ & $\mathbf{2 2}$ \\
& Purposive & 18 & 19 \\
$(0,2)$ & Random & $\mathbf{4 1}$ & $\mathbf{2 5}$ \\
& Purposive & 28 & 24 \\
$(0,3)$ & Random & $\mathbf{4 9}$ & $\mathbf{1 9}$ \\
& Purposive & 45 & 17 \\
$(1,0)$ & Random & 45 & 15 \\
& Purposive & $\mathbf{5 4}$ & $\mathbf{1 7}$ \\
$(1,1)$ & Random & 46 & 14 \\
& Purposive & $\mathbf{5 0}$ & $\mathbf{1 6}$ \\
$(1,2)$ & Random & $\mathbf{5 4}$ & $\mathbf{1 2}$ \\
& Purposive & 54 & 11 \\
$(1,3)$ & Random & 50 & 7 \\
& Purposive & $\mathbf{5 5}$ & $\mathbf{7}$ \\
$(2,0)$ & Random & 45 & 12 \\
& Purposive & $\mathbf{5 3}$ & $\mathbf{1 3}$ \\
$(2,1)$ & Random & $\mathbf{5 9}$ & $\mathbf{7}$ \\
$(2,2)$ & Purposive & 53 & 7 \\
& Random & 31 & $\mathbf{7}$ \\
$(2,3)$ & Purposive & $\mathbf{3 3}$ & 6 \\
& Random & $\mathbf{4 3}$ & $\mathbf{3}$ \\
\hline
\end{tabular}

$\epsilon=0.001$. SC measure underlines the performance of the model with $\epsilon=0.001$ in approximating/finding Pareto frontier. Additionally, in 2 instances, the model with $\epsilon=0.1$ was able to find a dominating solution to Pareto approximate solutions of the model with $\epsilon=0.001$. The model with $\epsilon=0.01$ was able to find a dominating solution to Pareto approximate solutions of the model with $\epsilon=0.001$ in 4 instances. These results emerge from the solutions found with $\epsilon=0.001$ not being optimal (See Corollary 1.). As having $\epsilon=0.001$ provides a better Pareto front approximation, heuristics' performances will be examined with respect to the model with $\epsilon=0.001$ in the sequel.

In 9 out of 11 instances, model performance is better than both heuristic methodologies when we consider set coverage measure. Additionally, in 2 instances, the random and purposive heuristic methodologies were able to find a dominating solution to Pareto approximate solutions of the model. When heuristic methodologies are compared with respect to each other, on average $16 \%$ of the points obtained with the purposive heuristic can dominate the points obtained with the random heuristics whereas $67 \%$ of the points obtained with the purposive heuristics can be dominated by the random heuristics. Moreover, in 4 out of 11 instances, the purposive heuristic methodology was able to find a dominating solution to Pareto approximate solutions of the random heuristics.

Table 10 provides a more detailed analysis on the performance of the heuristic solution methodologies compared to the mathematical model with $\epsilon=0.001$. With respect to the spacing measure, model performance is better in all instances, meaning that solution set of the model is more diverse. If we consider maximum spread measure, in 9 of the instances, the heuristic methods yield a larger value, that is, heuristics were able to capture a larger spectrum of the Pareto front. Additionally, in 6 of those instances, the purposive heuristic outperforms both the model and the random heuristic. Only in 3 of the instances, the random heuristic was better in terms of covering a larger spectrum of the Pareto front. Furthermore, the Pareto front spectrum covered per non-dominated solution is larger in all heuristic instances and in 9 out of 11 instances, the purposive heuristic captures a larger spectrum with a non-dominated solution. The reason for such an outcome can be attributed to both its performance with respect to maximum spread metric and having a fewer number of points generated by the method. Hence, it can be asserted that purposive improvement heuristic captures the Pareto front with less number of points to approximate the Pareto front.

In overall, the performance of the heuristic solution methodologies are better than the model under $\epsilon=0.001$ in terms of maximum spread, average maximum spread and computation time in comparison to mathematical model under $\epsilon=0.001$; however, in terms of spacing and set coverage heuristics are not performing well. The purposive improvement heuristic methodology performs better compared the random improvement heuristics when maximum spread and average maximum spread measures are considered; however, when set coverage performances of two heuristics are compared, random improvement heuristic outperforms the purposive improvement heuristic. This result can be attributed to the purposive heuristics being stuck in local optima and generating a fewer number of points.

Another analysis can be made based on how much the improvement methods improve the solution obtained in the construction phase in terms of both arc and node objectives. Table 11 depicts the improvement percentages of both of the heuristics in both objectives. The highest percentages are highlighted in the Table 11. It can be observed that random improvement heuristic improves arc and node objective up to 59\% and 25\%, respectively. Purposive improvement heuristic, on the other hand, contributes to improvement at most $55 \%$ in arc objective and $24 \%$ in node objective. The random improvement heuristic is more successful than the purposive improvement heuristic in finding corners of the Pareto front most of 
the time. Moreover, average improvement percentages underpin the success of random improvement heuristics; however, the difference in percentages is not that significant. On average, the random improvement heuristic can improve arc objective $43.9 \%$, node objective $12.9 \%$; on the other hand, these improvement percentages become $42.6 \%$ and $12.5 \%$, respectively, with the purposive improvement heuristic.

As discussed via Table 5-11, the random and purposive improvement heuristics have really good performance in terms of the computational time, maximum spread and average maximum spread. Moreover, random improvement heuristic has an advantage over the purposive improvement heuristic by providing larger improvements in both objectives and performing better than purposive improvement heuristic with respect to the set coverage metric. Hence, considering the computational requirements, improvement in both objectives and the performance with respect to set coverage, maximum and average maximum spread metrics, random improvement heuristic is more suitable than the other methodologies. With respect to spacing and set coverage metric, the mathematical model under $\epsilon=0.001$ dominates the other methodologies; however, the model's computational time requirement remains as a challenge.

\section{Extensions of PDARP}

In the scope of this paper, the PDARP and its heuristic methodologies are considered to facilitate early assessment of the disaster-affected region. Several extensions of the proposed model that incorporates assessment times into the model can be developed. Those extensions can be considered as a detailed assessment of the disaster-affected region where these assessments may require spending a certain amount of time on the node/arc being assessed. The proposed model in this study can be modified to capture the need to spend a certain amount of time in the node/arc being assessed. We refer new formulation as Post-disaster Assessment Routing with Assessment Time Problem (PDARATP). The following additional parameters and decision variables need to be defined for the PDARATP.

Additional Parameters:

$$
\kappa_{i} \quad \text { : time spent for assessing node } i \in N \text {. }
$$

$\lambda_{i j} \quad$ : time spent for assessing arc $(i, j) \in A$.

Additional Decision Variables:

$Y_{i k}^{\prime}: \begin{cases}1, & \text { if vehicle } k \text { monitors node } i \in N, \\ 0, & \text { otherwise. }\end{cases}$

$Z_{i j k}^{\prime}: \begin{cases}1, & \text { if vehicle } k \text { monitors arc }(i, j) \in A, \\ 0, & \text { otherwise. }\end{cases}$

The following mixed integer non-linear program for PDARATP can now be proposed:

$$
\begin{aligned}
& \text { maximize } f 1, f 2 \\
& \text { subject to }
\end{aligned}
$$

(1) $-(13)$

(21) $-(23)$

$Y_{j k}^{\prime} \leq \sum_{i \in N} X_{i j k} \quad \forall j \in N, \forall k \in M$

$Y_{j k}^{\prime} \leq \sum_{i \in N} \sum_{l \in N} a_{i l}^{j} \cdot X_{i l k} \quad \forall j \in N, \forall k \in D$

$Y_{j k}^{\prime} \geq \frac{1}{2} \cdot X_{i j k} \quad \forall(i, j) \in A, \forall k \in M$

$Y_{j k}^{\prime} \geq a_{i l}^{j} \cdot \frac{1}{2} \cdot X_{i l k} \quad \forall(i, l) \in A, \forall j \in N, \forall k \in D$

$Z_{i j k}^{\prime} \leq\left(X_{i j k}+X_{j i k}\right) \quad \forall(i, j),(j, i) \in A, \forall k \in M$

$Z_{i j k}^{\prime} \leq \sum_{(l, m) \in A}\left(b_{l m}^{i j} \cdot X_{l m k}\right)+\sum_{(m, l) \in A}\left(b_{m l}^{i j} \cdot X_{m l k}\right) \quad \forall(i, j) \in A, \forall k \in D$

$Z_{i j k}^{\prime} \geq \frac{1}{2 \cdot 2} \cdot\left(X_{i j k}+X_{j i k}\right) \quad \forall(i, j),(j, i) \in A \forall k \in M$

$Z_{i j k}^{\prime} \geq \frac{1}{2 \cdot 2} \cdot\left(b_{l m}^{i j} \cdot X_{l m k}+b_{m l}^{i j} \cdot X_{m l k}\right) \quad \forall(i, j),(l, m),(m, l) \in A, \forall k \in D$ 


$$
\begin{aligned}
& \sum_{(i, j) \in A}\left(\frac{d_{i j}}{v} \cdot X_{i j k}+\lambda_{i j} \cdot Z_{i j k}^{\prime}\right)+\sum_{i \in N} \kappa_{i} \cdot Y_{i k}^{\prime} \leq T \quad \forall k \in V \\
& \sum_{j \in N}\left(u_{i j k}-u_{j i k}\right)-\left(\sum_{j \in N} \frac{d_{i j}}{v} \cdot X_{i j k}+\lambda_{i j} \cdot Z_{i j k}^{\prime}+\kappa_{j} \cdot Y_{j k}^{\prime}\right)=0 \quad \forall i \in N \backslash\{s\}, \forall k \in V \\
& u_{s j k}=\frac{d_{s j}}{v} \cdot X_{s j k}+\lambda_{s j} \cdot Z_{s j k}^{\prime}+\kappa_{j} \cdot Y_{j k}^{\prime} \quad \forall j \in N \backslash\{s\}, \forall k \in V \\
& u_{i s k} \leq T \cdot X_{i s k} \quad \forall i \in N \backslash\{s\}, \forall k \in V \quad \forall(i, j) \in A, j \neq s, \forall k \in V \\
& u_{i j k} \leq\left(T-\frac{d_{j s}}{v}\right) \cdot X_{i j k} \quad \forall(i, j) \in A, j \neq s, \forall k \in V \\
& u_{i j k} \leq \max \left\{T-\frac{d_{j s}}{v}, 0\right\} \quad \forall(i, j) \in A, i \neq s, \forall k \in V \\
& u_{i j k} \geq \frac{\left(d_{s i}+d_{i j}\right)}{2 \cdot v} \cdot X_{i j k}+\kappa_{s} \cdot Y_{s k}^{\prime}+\kappa_{j} \cdot Y_{j k}^{\prime}+\lambda_{i j} \cdot Z_{i j k}^{\prime} \\
& Z_{i j}^{k} \in\{0,1\}, \quad \forall(i, j) \in A, \forall k \in V \\
& Y_{j}^{k} \in\{0,1\}, \quad \forall j \in N \forall k \in V
\end{aligned}
$$

The problem has a similar structure as PDARP; however, in order to incorporate spending certain amount of time for assessment, we need to detect which vehicle is responsible from the assessment of which nodes/arcs. Constraints (30)-(33) monitor the assessment of node $j$ by the vehicle $k$. Constraints (30) and (31) are similar to constraint (6). As the new decision variable, $Y_{i k}^{\prime}$ takes vehicle index into account, the constraint (6) is separated to capture which vehicle conducts the assessment of node $i$. Constraints (32) and (33) are similar to constraints (7) and (8). Constraints (34)-(37) check if arc (i,j) is monitored by the vehicle $k$ in either direction. Constraints (34) and (35) are a version of the constraint (9) without summation over vehicle sets. Constraints (36) and (37) are similar to constraints (10) and (11), only difference is that the left hand side of the constraints bear a vehicle index for arc assessment. Total time bound is given by the constraint (38) which is similar to the constraint (14). Constraint (39) ensures the connectivity of the tour for each vehicle k considering assessment time requirements. In parallel to constraint (16), constraint (40) assigns the time spent by leaving the depot but adds the assessment time of nodes/arcs on the way to the connectivity variable. By constraints (41)-(43), an upper bound on connectivity variable is imposed. Those three constraints are indeed a version of (17)-(19) where connectivity variable is time-based. By constraint (44), we ensure that connectivity variable takes a positive value when a vehicle traverses that particular network element. Therefore, disconnected tours are eliminated via constraints (39)-(44).

To test the developed mathematical model, we used the dataset Kartal as discussed in Section 5.1 (Kilci et al., 2018). Test parameter specifications of the PDARP are still valid for this problem. However, for this problem setting, we only consider 1 drone and 1 motorcycle. Additional parameters, $\kappa$, and $\lambda$ that represent the time required for assessment, are defined based on node weights and distance values, respectively. $\kappa$ values assigned to the nodes in a way that node with the highest node weight gets 10 minutes for assessment and the rest are assigned proportionally to their weight values. $\lambda$ is defined as $10 \%$ of the travel time of the particular arc.

The performances of the models are presented in Table 12. As the new model allocate an extra time for the assessment efforts, it is expected that the number of population points and the road segments assessed will decline. It is observed that when certain assessment times introduced for the network elements, in first two hours in the disaster aftermath approximately $36 \%$ of the time, 43 minutes, spent solely on assessing network elements, while the rest is spent on traversal and the assessment. As two new binary variables are introduced to the problem and they appear in connectivity constraints, PDARATP is computationally more challenging. Total solution time per Pareto solution and the average gap values reported in Table 12.

Since first hours in the disaster aftermath bear a great importance on the alleviation of the human suffering, one might consider PDARP as an immediate assessment strategy and PDARATP as a more detailed assessment operation following the PDARP. The reasons for proposing PDARP as an immediate assessment strategy are PDARP's ability to evaluate disaster impact on more network elements (population points and road segments) and PDARP enables a disaster overview of a wider area. 
Table 12

Comparison of the models PDARP and PDARATP.

\begin{tabular}{lllll}
\hline$|D|$ & $|M|$ & Criteria & PDARP & PDARATP \\
\hline 0 & 1 & Average Node objective & 6.053 & 2.806 \\
& & Average Arc Objective & 5.328 & 3.675 \\
& & Number of Pareto Solutions & 11 & 1 \\
& & Total Solution Time per Pareto Solution & 0.05 & 0.08 \\
& & Average Gap & 0.00 & 0.00 \\
& & Required Assessment Time over Route Duration & - & 36 \\
1 & \multirow{2}{*}{0} & Average Node Objective & 7.536 & 2.893 \\
& & Average Arc Objective & 7.050 & 4.299 \\
& & Number of Pareto Solutions & 19 & 1 \\
& & Total Solution Time per Pareto Solution & 1.66 & 1.74 \\
& Average Gap & 0.06 & 0.00 \\
& & Required Assessment Time over Route Duration & - & 36 \\
1 & \multirow{2}{*}{1} & Average Node Objective & 8.324 & 5.279 \\
& Average Arc Objective & 11.736 & 6.389 \\
& Number of Pareto Solutions & 22 & 4 \\
& Total Solution Time per Pareto Solution & 0.94 & 6.00 \\
& Average Gap & 0.00 & 9.14 \\
& Required Assessment Time over Route Duration & - & 35 \\
\hline
\end{tabular}

\section{Managerial and policy implications}

In this study, the post-disaster assessment strategy is developed as a tool to assist disaster relief operations by assessing the severity of the disaster and the urgency for relief. Several managerial insights provided by this research can be presented.

This study develops a model for the damage assessment in an aftermath of any disaster which focuses on population points and road segments. Damage information obtained from both elements translate into resolved uncertainties in three immediate logistics operations: evacuation, relief item distribution, and debris removal. The consecutive operations may perform poorly with the unresolved uncertainties in the demand. Thus, post-disaster assessments can facilitate more timely relief by conducting early assessments to determine the severity of the disaster impact and urgency of the need for relief. Hence, by assessing damage, disaster managers can distribute relief items and canalize their relief operations to the point where they are needed most.

Assessment problems studied in the literature largely focus on needs assessment in population points. In this study, with having capable resources at hand, we observe that it is possible to conduct an assessment in not only population points but also on the road segments. To reach disaster victims, evacuate them from the affected region or supply them with relief items in a short period, the situation of the transportation network should also be known. Also, in accordance with the overview of the disaster assessment, disaster management authorities can plan debris removals in parallel to relief routing operations in order to reach disaster victims in a timely manner.

The proposed model in this study presents a way of narrowing down possible performance deficiencies in the response phase operations through resolving uncertainties in the disaster aftermath. With preparing and implementing the postdisaster assessment strategy, the disaster management authorities could produce more efficient responses; thus, mitigates the pain and suffering of disaster victims, as early as possible.

\section{Conclusion}

Due to the importance of information in the post-disaster response phase, this study focuses on the damage assessment process in an aftermath of a disaster and introduces a new problem that facilitates effective information gathering on population centers (nodes) and road segments (arcs) through deciding on assessment team routes.

In the literature, there is a limited number of studies that focus on assessment operations in the aftermath of a disaster. Existing studies are solely focused on assessment of population points. To the best of our knowledge, there is no study that considers assessment in both elements; although transportation network condition directly influences evacuation and relief item distribution operations.

With this study, we highlight the importance of considering both network elements in the assessment stage and develop an appropriate assessment strategy. We develop a mathematical model that provides damage information in the affected region by considering both the importance of population centers and road segments on the transportation network through using aerial and ground vehicles (drones and motorcycles). To assist post-disaster response phase operations by obtaining information about the extent of damage in the area in a short period, a completion deadline is imposed via route duration constraints. Additionally, as opposed to standard vehicle routing problems, we let population points to be visited multiple times to better capture the disaster impact on road segments.

The problem characteristics imply a general routing aspect which combines assessment of critical population centers (nodes), and road segments (arcs). However, as the assessment operation is focused on two distinct network elements, a 
bi-objective approach to the problem is required. For this reason, we studied relief routing, multi-objective routing and general routing problems in the literature with its variants. Then, we mathematically modeled bi-objective general routing with profits problem considering arc and node profits as separate objectives. Bi-objectivity of the problem is handled with $\epsilon$-constraint method. The model returns assessment routes for vehicles where arcs and nodes to traverse are selected. Additionally, the use of aerial vehicles contributes to the assessment of a possibly large number of arcs and nodes due to its angular point of view. We present a heuristic solution methodology to solve the PDARP. Our computational results show that the proposed algorithm can find a high-quality approximation of Pareto front for the PDARP that mitigates the solution time difficulties.

As damage assessment operations have not received much attention in humanitarian logistics or in OR literature yet, there can be several research directions that focus on damage assessments in disaster aftermath. First, although this study presents some results suggesting that the proposed heuristic methodologies may attain good solutions as it provides an approximation of Pareto front in a short processing time, developing solution methodologies that can provide exact Pareto fronts or better Pareto front approximation would be valuable to obtain better benchmark solutions to evaluate the performance of the heuristics.

Future research direction can be related to characterizing the post-disaster uncertainties in developing an assessment plan. Our study considers disaster network as an off-road network where off-road motorcycles are utilized with a certain average speed; however, in the aftermath of a disaster, there can be some road segments which are severely disrupted and some with little to no damage. Hence, it would be valuable to incorporate travel time uncertainties and terrain conditions in routing decisions. Also, uncertainties may arise during the assessment process. Although the proposed model in this study is modified to spend a certain amount of time in the node/arc being assessed, the node/arc assessment uncertainties cannot be captured with the current model.

In the proposed problem, we assumed cost is a linear function of the distance and we imposed a distance bound on the total route duration. This can be considered as having a budget which is in line with many humanitarian research papers. However, of course, cost concerns can also be incorporated into the model as a third objective which may include the vehicle and transportation costs.

Additionally, the current study considers information on the disaster impact is made available once the assessment vehicles return to the depot (disaster management center). This can be counted as a valid assumption considering the disruptions can occur in the information transmission infrastructure as a result of a disaster. However, one can consider relaxing this assumption and incorporate spatial-temporal uncertainties that arise when the drones and motorcycles are conducting assessments in collaboration given that the drones angular field of view can assist the motorcycle in assessment. We hope that the model and algorithms proposed in this study will constitute a new angle for future research that considers further complexities in decision making for post-disaster damage assessments.

\section{Appendix A. Illustration of the Constructive Heuristic Algorithm}

For example, suppose we have a disaster network as demonstrated in the Fig. A.7 and 1 drone to be utilized in assessment. On a given network, it is important to note that fourth node can be assessed by flying over arc $(3,9)$ and $(9,3)$.

Assume that the profit is defined by the value added by traversing a node per distance travelled and there is a distance limit for the drone, and it is 20 . We first start by evaluating the shortest paths of each node pairs. Then, among the feasible paths (a vehicle can complete its tour within the distance limit), the one which yields the highest node profit per distance

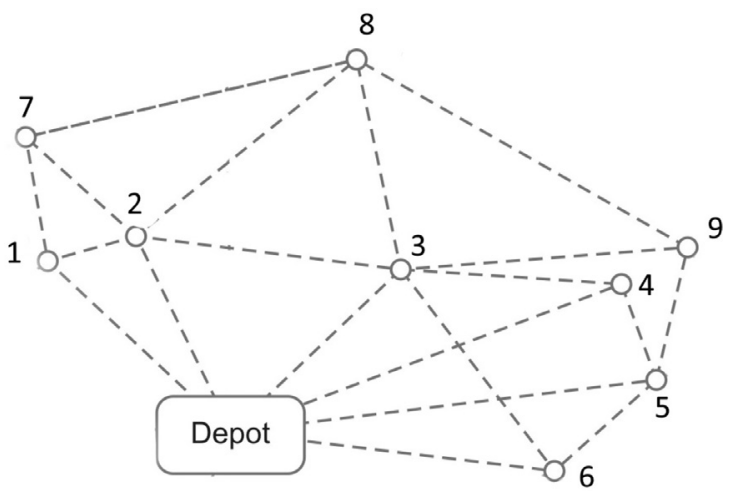

Fig. A.7. An example disaster network diagram. 
Table A.13

Shortest paths from the depot node to other nodes.

\begin{tabular}{lll}
\hline From & To & Shortest Path \\
\hline 0 & 1 & {$\left[\begin{array}{ll}0 & 1\end{array}\right]$} \\
0 & 2 & {$\left[\begin{array}{ll}0 & 2\end{array}\right]$} \\
0 & 3 & {$\left[\begin{array}{ll}0 & 3\end{array}\right]$} \\
0 & 4 & {$\left[\begin{array}{ll}0 & 4\end{array}\right]$} \\
0 & 5 & {$\left[\begin{array}{ll}0 & 5\end{array}\right]$} \\
0 & 6 & {$\left[\begin{array}{ll}0 & 6\end{array}\right]$} \\
0 & 7 & {$\left[\begin{array}{ll}0 & 2\end{array}\right]$} \\
0 & 8 & {$\left[\begin{array}{lll}0 & 3 & 8\end{array}\right]$} \\
0 & 9 & {$\left[\begin{array}{lll}0 & 3 & 9\end{array}\right]$} \\
\hline
\end{tabular}

\section{Table A.14}

Shortest paths from the node 3 to other nodes.

\begin{tabular}{|c|c|c|}
\hline From & To & Shortest Path \\
\hline 3 & 0 & {$\left[\begin{array}{ll}3 & 0\end{array}\right]$} \\
\hline 3 & 1 & {$\left[\begin{array}{lll}3 & 2 & 1\end{array}\right]$} \\
\hline 3 & 2 & {$\left[\begin{array}{ll}3 & 2\end{array}\right]$} \\
\hline 3 & 4 & {$\left[\begin{array}{ll}3 & 4\end{array}\right]$} \\
\hline 3 & 5 & {$\left[\begin{array}{lll}3 & 4 & 5\end{array}\right]$} \\
\hline 3 & 6 & {$\left[\begin{array}{ll}3 & 6\end{array}\right]$} \\
\hline 3 & 7 & {$\left[\begin{array}{lll}3 & 2 & 7\end{array}\right]$} \\
\hline 3 & 8 & {$\left[\begin{array}{ll}3 & 8\end{array}\right]$} \\
\hline 3 & 9 & [3 9] \\
\hline
\end{tabular}

is added to the path. Let shortest path distances of the network be:

$\left[\begin{array}{cccccccccc}0.0 & 3.7 & 3.2 & 3.4 & 6.2 & 6.3 & 4.8 & 5.4 & 6.5 & 7.6 \\ 3.7 & 0.0 & 1.4 & 5.3 & 8.5 & 9.9 & 8.5 & 1.9 & 5.5 & 9.5 \\ 3.2 & 1.4 & 0.0 & 3.9 & 7.1 & 8.5 & 7.6 & 2.2 & 4.1 & 8.1 \\ 3.4 & 5.3 & 3.9 & 0.0 & 3.2 & 4.6 & 3.7 & 6.1 & 3.1 & 4.2 \\ 6.2 & 8.5 & 7.1 & 3.2 & 0.0 & 1.4 & 3.4 & 9.3 & 6.3 & 3.5 \\ 6.3 & 9.9 & 8.5 & 4.6 & 1.4 & 0.0 & 2.0 & 10.7 & 7.7 & 2.1 \\ 4.8 & 8.5 & 7.6 & 3.7 & 3.4 & 2.0 & 0.0 & 9.8 & 6.8 & 4.1 \\ 5.4 & 1.9 & 2.2 & 6.1 & 9.3 & 10.7 & 9.8 & 0.0 & 5.1 & 10.3 \\ 6.5 & 5.5 & 4.1 & 3.1 & 6.3 & 7.7 & 6.8 & 5.1 & 0.0 & 5.7 \\ 7.6 & 9.5 & 8.1 & 4.2 & 3.5 & 2.1 & 4.1 & 10.3 & 5.7 & 0.0\end{array}\right]$

In the Table A.13, the shortest paths emerging from the depot node to other nodes are indicated.

Moreover, let the following vector to be node weights of the corresponding nodes.

$$
\left[\begin{array}{llllllllll}
0.5 & 0.2 & 0.7 & 0.3 & 0.5 & 1.0 & 0.1 & 0.1 & 0.5 & 0.5
\end{array}\right]
$$

It is observed that there is at least one node where a drone can go and come back to the depot. For each path emerging from the depot node to other nodes, profits that can be collected are calculated. That is, values of nodes on the path divided by the shortest path distance.

$$
\left[\begin{array}{llllllllll}
- & 0.189 & 0.281 & 0.294 & 0.129 & 0.238 & 0.229 & 0.241 & 0.200 & 0.237
\end{array}\right]
$$

Since the path from the depot node, 0 , to the node 3 yields the highest profit, we add $[0,3]$ to the path. Total distance traveled becomes 3.4. Then, from the node 3, we evaluate the profits of the feasible paths. In the Table A.14, the shortest paths from the node 3 to other nodes are indicated.

For each path emerging from the node 3 to the other nodes, profits that can be collected are calculated based on the profit definition. That is, values of not assessed nodes on the path divided by the shortest path distance.

$$
\left[\begin{array}{llllllllll}
0.000 & 0.038 & 0.179 & - & 0.156 & 0.326 & 0.027 & 0.131 & 0.161 & 0.238
\end{array}\right]
$$

As the path from the node 3 to the node 5 yields the highest profit and it does not violate the total distance constraint, it is considered as a candidate. Total tour distance of $[0,3,4,5,0]$ is 14.3 . Hence we add $[3,4,5]$ to the path. Total distance travelled becomes 8.0. Then, from node 5 we evaluate the profits of the feasible paths. In the Table A.15, the shortest paths from the node 5 to other nodes are indicated.

For each path emerging from the node 5 to the other nodes, profits that can be collected are calculated based on the profit definition. That is, values of not assessed nodes on the path divided by the shortest path distance.

$$
\left[\begin{array}{llllllllll}
0.000 & 0.091 & 0.082 & 0.000 & 0.000 & - & 0.050 & 0.075 & 0.065 & 0.238
\end{array}\right]
$$


Table A.15

Shortest paths from the node 5 to other nodes.

\begin{tabular}{|c|c|c|}
\hline From & To & Shortest Path \\
\hline 5 & 0 & {$\left[\begin{array}{ll}5 & 0\end{array}\right]$} \\
\hline 5 & 1 & {$\left[\begin{array}{lllll}5 & 4 & 3 & 2 & 1\end{array}\right]$} \\
\hline 5 & 2 & {$\left[\begin{array}{llll}5 & 4 & 3 & 2\end{array}\right]$} \\
\hline 5 & 3 & {$\left[\begin{array}{lll}5 & 4 & 3\end{array}\right]$} \\
\hline 5 & 4 & {$\left[\begin{array}{ll}5 & 4\end{array}\right]$} \\
\hline 5 & 6 & {$\left[\begin{array}{ll}5 & 6\end{array}\right]$} \\
\hline 5 & 7 & {$\left[\begin{array}{lllll}5 & 4 & 3 & 2 & 7\end{array}\right]$} \\
\hline 5 & 8 & {$\left[\begin{array}{llll}5 & 4 & 3 & 8\end{array}\right]$} \\
\hline 5 & 9 & [5 9] \\
\hline
\end{tabular}

Some of the paths are now infeasible. Those are the paths going to the nodes 2, 7, and 8 . Among the feasible paths, the path, from the node 5 to the node 9 yields the highest profit, and total tour distance of $[0,3,4,5,9,0]$ is 17.7 . Hence, we append path $[5,9]$ to the path. Total distance travelled becomes 10.1 . Then, from the node 9 , we evaluate the profits of the feasible paths. The constructive algorithm procedure continues in this fashion, at each step, we aim to guarantee a vehicle can return to the depot. In this example, we used the node weight collected over distance traveled as the profit definition; however, the other definitions discussed in Section 4.1 can also be used.

\section{References}

Aeromao, 2014. Aeromapper talon - aeromao- uav systems. http://www.aeromao.com/aeromapper_talon_uav/. (Accessed on 11/23/2017).

Agatz, N., Bouman, P., Schmidt, M., 2016. Optimization Approaches for the Traveling Salesman Problem with Drone. ERIM Report Series Research in Management. Erasmus Research Institute of Management (ERIM), ERIM is the joint research institute of the Rotterdam School of Management, Erasmus University and the Erasmus School of Economics (ESE) at Erasmus University Rotterdam.

Akbari, V., Salman, F.S., 2017. Multi-vehicle synchronized arc routing problem to restore post-disaster network connectivity. Eur. J. Oper. Res. 257(2), $625-640$.

Altay, N., Green, W., 2006. Or/ms research in disaster operations management. Eur. J. Oper. Res. 175, $475-493$.

Amazon, 2016. Amazon prime air. https://www.amazon.com/b?node=8037720011. (Accessed on 11/19/2017)

Aráoz, J., Fernández, E., Zoltan, C., 2006. Privatized rural postman problem. Comput. Oper. Res. 33, 3432-3449.

Archetti, C., Bertazzi, L., Lagana, D., Vocaturo, F., 2017. The undirected capacitated general routing problem with profits. Eur. J. Oper. Res. $257,822-833$.

Archetti, C., Feillet, D., Hertz, A., Speranza, M., 2009. The capacitated team orienteering and profitable tour problems. J. Oper. Res. Soc. 60, 831-842.

Archetti, C., Feillet, D., Hertz, A., Speranza, M.G., 2010. The undirected capacitated arc routing problem with profits. Comput. Oper. Res. 37, 1860-1869.

Archetti, C., Speranza, M.G., Corberán, A., José, M. Sanchis, I.P., 2014. The team orienteering arc routing problem. Transp. Sci. 48, 442-457.

Awerbuch, B., Azar, Y., Blum, A., Vempala, S., 1998. New approximation guarantees for minimum-weight k-trees and prize collecting travelling salesmen. SIAM J. Comput. 28, 254-262.

Balas, E., 1989. The prize collecting traveling salesman problem. Networks 19, 621-636.

Balcik, B., 2017. Site selection and vehicle routing for post-disaster rapid needs assessment. Transp. Res. Part E 101, 30-58. doi:10.1016/j.tre.2017.01.002.

Bayram, V., Yaman, H., 2015. A stochastic programming approach for shelter location and evacuation planning. Optim. Online 2015-09-5088.

Berktas, N., Kara, B.Y., Karasan, O.E., 2016. Solution methodologies for debris removal in disaster response. EURO J. Comput. Optim. 4, $403-445$.

Butt, S., Cavalier, T., 1994. A heuristic for the multiple tour maximum collection problem. Comput. Oper. Res. 21, 101-111.

Camacho-Vallejo, J.-F., Gonzalez-Rodriguez, E., Almaguer, F.-J., Gonzalez-Ramirez, R.G., 2015. A bi-level optimization model for aid distribution after the occurrence of a disaster. J. Clean. Prod. 105, 134-145.

Campbell, A.M., Vandenbussche, D., Hermann, W., 2008. Routing for relief efforts. Transportation Science 42(2), 127-145.

Caunhye, A.M., Nie, X., Pokharel, S., 2012. Optimization models in emergency logistics: a literature review. Socio. Econ. Planning Sci. 46, 4-13.

Celik, M., Ergun, O., Johnson, B., Keskinocak, P., Lorca, A., Pekgun, P., Swann, J., 2012. Humanitarian logistics. In: INFORMS Tutorials in Operations Research: New Directions in Informatics, Optimization, Logistics, and Production. INFORMS, USA, pp. 18-49.

Celik, M., Ergun, O., Keskinocak, P., 2015. The post-disaster debris clearance problem under incomplete information. Oper. Res. 63 (1), 65-85. doi:10.1287/ opre.2014.1342.

Chao, I.-M., Golden, B., Wasil, E., 1996. The team orienteering problem. Eur. J. Oper. Res. 8, 464-474.

Choi-Fitzpatrick, A., Chavarria, D., Cychosz, E., Dingens, J.P., Duffey, M., Koebel, K., Siriphanh, S., Yurika Tulen, M., Watanabe, H., Juskauskas, T., et al., 2016. Up in the air: a global estimate of non-violent drone use 2009-2015. Up in the Air: A Global Estimate of Non-Violent Drone Use $2009-2015$ 1.

Chou, T., Yeh, M.L., Chen, Y., Chen, Y., 2010. Disaster monitoring and management by the unmanned aerial vehicle technology. In: Wagner W., Székely, B. (eds.): ISPRS TC VII Symposium - 100 Years ISPRS, Vienna, Austria, IAPRS, Part 7B 36, 137-142.

Daley, W., Karpati, A., Sheik, M., 2001. Needs assessment of the displaced population following the august 1999 earthquake in turkey. Disasters 25, 67-75.

Dantzig, G., Fulkerson, R., Johnson, S., 1954. Solution of a large-scale traveling-salesman problem. J. Oper. Res. Soc. Am. 2, 393-410.

Dantzig, G., Ramser, J., 1959. The truck dispatching problem. Manage. Sci. 6, 80-91.

Deitch, R., Ladany, S., 2000. The one-period bus touring problem: solved by an effective heuristic for the orienteering tour problem and improvement algorithm. Eur. J. Oper. Res. 127, 69-77.

Dell'Amico, M., Maffioli, F., Värbrand, P., 1995. On prize-collecting tours and the asymmetric travelling salesman problem. Int. Trans. Oper. Res. 2 (3), 297-308.

DHL, 2014. Dhl-press release-english. http://www.dhl.com/en/press/releases/releases_2014/group/dhl_parcelcopter_launches_initial_operations_for_ research purposes.html. (Accessed on 11/19/2017).

Feillet, D., Dejax, P., Gendreau, M., 2005. Travelling salesman problems with profits. Transp. Sci. 39, $188-205$.

Galindo, G., Batta, R., 2013. Review of recent developments in or/ms research in disaster operations management. Eur. J Oper. Res. 230, 201-211.

Giordan, D., Manconi, A., Remondino, F., Nex, F., 2017. Use of unmanned aerial vehicles in monitoring application and management of natural hazards. Geomatics, Natural Hazards Risk 8 (1), 1-4. doi:10.1080/19475705.2017.1315619.

Golden, B., Levy, L., Dahl, R., 1981. Two generalizations of the travelling salesman problem. Manage. Sci. 9, $201-205$.

Google, 2014. Google tests drone deliveries in project wing trials - BBC news. http://www.bbc.com/news/technology-28964260. (Accessed on 11/19/2017).

Guan, M., 1962. Graphic programming using odd or even points. Chinese Math. 1, 273-277.

Ha, Q.M., Deville, Y., Pham, Q.D., Hà, M.H., 2018. On the min-cost traveling salesman problem with drone. Transp. Res. Part C 86, 597-621. 
Haarbrink, R., Koers, E., 2006. Helicopter uav for photogrammetry and rapid response. Inter-Commission WG I/V, Autonomous Navigation.

Houming, F., Tong, Z., Xiaoyan, Z., Mingbao, J., Guosong, D., 2008. Research on emergency relief goods distribution after regional natural disaster occurring. International Conference on Information Management, Innovation Management and Industrial Engineering.

Hua, Z.-H., Sheu, J.-B., 2013. Post-disaster debris reverse logistics management under psychological cost minimization. Transp. Res. Part B 55, 118-141.

Huang, H., Long, J., Yi, W., Yi, Q., Zhang, G., Lei, B., 2017. A method for using unmanned aerial vehicles for emergency investigation of single geo-hazards and sample applications of this method. Nat. Hazards Earth Syst. Sci. 17 (11), 1961-1979. doi:10.5194/nhess-17-1961-2017.

Huang, M., Smilowitz, K., Balcik, B., 2013. A continuous approximation approach for assessment routing in disaster relief. Transp. Res. Part B 50, 20-41.

Hyun, C.J., Kim, Y., Kim, Y.K., 1998. A genetic algorithm for multiple objective sequencing problems in mixed model assembly lines. Comput. Oper. Res. 25 (7), 675-690. doi:10.1016/S0305-0548(98)00026-4.

IFRC, 2016. World disasters report 2016 - international federation of red cross and red crescent societies. http://media.ifrc.org/ifrc/publications/ world-disasters-report-2016/. (Accessed on 09/30/2017).

Johnson, M., Wilfert, R., 2008. Rapid needs assessments and gis. FOCUS Field Epidemiol. 5, 1-5.

Jozefowiez, N., Semet, F., Talbi, E.-G., 2008. Multi-objective vehicle routing problems. Eur. J. Oper. Res. 189, $293-309$.

Kara, B.Y., Savaser, S., 2017. Humanitarian Logistics. INFORMS, pp. 263-303.

Kataoka, S., Morito, S., 1988. An algorithm for single constraint maximum collection problem. J. Oper. Res. Soc. Japan 31, 515-530.

Kilci, F., Bozkaya, B., Kara, B. Y., Oruc, B. E., 2018. Kartal district of Istanbul. Mendeley Data. V1, https://doi.org/10.17632/xhz8m74pp7.1.

Kilci, F., Kara, B.Y., Bozkaya, B., 2015. Locating temporary shelter areas after an earthquake: A case for Turkey. Eur. J. Oper. Res. 243(1), 323-332.

Kovács, G., Spens, K., 2007. Humanitarian logistics in disaster relief operations. Int. J. Phys. Distribut. Logistics Manage. 37, 99-114.

Laporte, G., Martello, S., 1990. The selective travelling salesman problem. Discrete Appl. Math. 26, 193-207.

Long, D., 1997. Logistics for disaster relief: engineering on the run. IIE Solutions 29, 26-29.

Malandraki, C., Daskin, M.S., 1993. The maximum benefit chinese postman problem and the maximum benefit traveling salesman problem. Eur. J. Oper. Res. $65,218-234$

Molina, P., Colomina, I., Victoria, T., Skaloud, J., Kornus, W., Prades, R., Aguilera, C., 2012. Searching lost people with uavs: the system and results of the close-search project. Int. Arch. Photogrammetry Remote Sensing Spatial Inf. Sci. 39, 441-446.

Murray, C.C., Chu, A.G., 2015. The flying sidekick traveling salesman problem: optimization of drone-assisted parcel delivery. Transp. Res. Part C 54, 86-109. Nex, F., Remondino, F., 2014. Uav for 3d mapping applications: a review. Appl. Geomatics 6 (1), 1-15.

Orloff, C., 1974. A fundamental problem in vehicle routing. Networks 4, 35-64.

Ozdamar, L., 2011. Planning helicopter logistics in disaster relief. OR Spectrum 33(3), 655-672.

Ozdamar, L., Ertem, M., 2015. Models, solutions and enabling technologies in humanitarian logistics. Eur. J. Oper. Res. 244, 55-65.

Ozkapici, D.B., Ertem, M.A., Aygunes, H., 2016. Intermodal humanitarian logistics model based on maritime transportation in istanbul. Natural Hazards 83(1), 345-364.

Ramesh, R., Brown, K.M., 1991. An efficient four-phase heuristic for the generalized orienteering tour problem. Comput. Oper. Res. $18,151-165$.

Sahin, H., Kara, B.Y., Karasan, O.E., 2016. Debris removal during disaster response: a case for turkey. Socioecon. Plann. Sci. 53, 49-59.

Schott, J.R., 1995. Fault Tolerant Design Using Single and Multicriteria Genetic Algorithm Optimization. MASSACHUSETTS INSTITUTE OF TECHNOLOGY, Department of Aeronautics and Astronautics, Massachusetts Institute of Technology, Cambridge, Massachusetts. Masters thesis.

Scott, J., Scott, C., 2017. Drone delivery models for healthcare. In: Proceedings of the 50th Hawaii International Conference on System Sciences, pp. 3297-3304.

Souffriau, W., Vansteenwegen, P., Berghe, G.V., Oudheusde, D.V., 2011. The planning of cycle trips in the province of east flanders. OMEGA 39. 209-203

Tatham, P., 2009. An investigation into the suitability of the use of unmanned aerial vehicle systems (uavs) to support the initial needs assessment process in rapid onset humanitarian disasters. Int. J. Risk Assess. Manag. 13, 60-78.

Tsiligirides, T., 1984. Heuristic methods applied to orienteering. J. Oper. Res. Soc. 35, 797-809.

Tzeng, G.-H., Checg, H.-J., Huang, T.D., 2007. Multi-objective optimal planning for designing relief delivery systems. Transportation Research Part E: Logistics and Transportation Review 43(6), 673-686.

UAE, 2014. Uae to use drones for citizen services-news-al jazeera. http://www.aljazeera.com/news/middleeast/2014/02/uae-use-drones-governmentservices-20142121717319272.html. (Accessed on 11/19/2017).

Yan, S., Shih, Y.-L., 2009. Optimal scheduling of emergency roadway repair and subsequent relief distribution. Comput. Oper. Res. 36(6), $2049-2065$.

Zitzler, E., Thiele, L., 1998. Multiobjective optimization using evolutionary algorithms - A comparative case study. Springer Berlin Heidelberg, Berlin, Heidelberg, pp. 292-301. 